\title{
Survey of Compressions Observed in the Heliosheath with the Spacecraft Voyager
}

\author{
Danie Benjamín Berdichevsky¹,2 \\ ${ }^{1}$ IFIR/CONICET-UNR, Esmeralda y 27 de Febrero, Rosario, Santa Fe, RA \\ ${ }^{2}$ TRIDENT-BERDICHEVSKY, DANIEL B. Partnership, Greenbelt, MD, USA \\ Email: dbberdi@gmail.com
}

How to cite this paper: Berdichevsky, D.B. (2021) Survey of Compressions Observed in the Heliosheath with the Spacecraft Voyager. Advances in Aerospace Science and Technology, 6, 43-66.

https://doi.org/10.4236/aast.2021.61004

Received: November 17, 2020

Accepted: March 15, 2021

Published: March 18, 2021

Copyright $\odot 2021$ by author(s) and Scientific Research Publishing Inc. This work is licensed under the Creative Commons Attribution International License (CC BY 4.0).

http://creativecommons.org/licenses/by/4.0/

\begin{abstract}
Examples of changes in the magnitude of the B-field after the heliosphere termination shock (TS) with both Voyager spacecraft (SC) are presented. The work focuses on similarities and differences in the observations at their in-situ measurements along divergent paths. The presented results were collected where the accuracy of the magnetometer is the highest. These locations are those wherein, four to seven times during the year, the SC performs about 330 minutes of slow rotations identified in the SC language as MAGROLs. They are next reviewed, with the understanding that after the TS, at MAGROLs, the solar wind (SW) flows appear to be mostly sub-magnetosonic and compressional in this region, region named helio-sheath (HS). This is a preliminary survey that uses $48 \mathrm{sec}$ B-field averages. The time-intervals in this work fill gaps in the currently available studies for longer time intervals. The present study reinforces the view that in the HS after the TS the SW is most likely strongly compressional. Further we discuss the fact that observed fluctuation intensity-modes of the B-field in our time-ranges appear to be much more pronounced at the Voyager 2 path than at the Voyager 1 path.
\end{abstract}

\section{Keywords}

Magnetic Field Measurement Techniques, Solar Heliosphere's Heliosheath, MHD

\section{Introduction}

In our study using $48 \mathrm{~s}$ magnetic field density B (for a discussion of the physical meaning and the magnetic field density properties, and all the field of classical electrodynamics see e.g. [1]) we survey similarities and differences in the helio-sheath (HS) for intervals lasting from about $3 \mathrm{~min}$ to a few hours. In this way our study extends the thorough documentation of conditions [2] [3], which take 
place after termination of the SW supersonic flow, when each one of the two SCs of the VOYAGER mission transitioned to the sub-magnetosonic flow region, i.e. HS after the crossing of the "thin transition layer" known as the TS (For an overview of shocks in the heliosphere see e.g., [4] [5] [6]).

NSSDC/NASA provides the public/interested-worker for the following Voyager mission's available B magnetic field density: $48 \mathrm{sec}, 60 \mathrm{~min}$, daily averages (ave) as well as the highest readily available resolution of about $1.92 \mathrm{~s}$. It is possible to obtain $0.5 \mathrm{~s}$ ave, when available, through special agreement with the Voyager's magnetometer instrument production team.

It is well known that shocks in the SW, where the SW is a medium that possesses hydromagnetic (also commonly known as MHD or magnetohydrodynamics) properties, produce a transition from a dominated highly alfvenic MHD-state to a compressional state, see e.g. [7], where the traveling shock driven by solar coronal mass ejections is presented in their manifestations in radio signal emission, acceleration of energetic ions (with a moderately strong shock acceleration of electrons). [7] shows these features in its Figure 1 including a timeline for magnetic field and plasma SW in-situ measurements for about a month long period, belonging to the maximum of the Solar Cycle 23, see e.g. [8]. General characteristics of shocks in the HS observed by the SC Wind are discussed in [5] and literature therein. The particular nature of the proton foreshock of the well-known Earth's magnetosphere-SW shock is discussed in [4] and also in the literature therein. As such it is not surprising that we find strong compressional features in the HS after the TS. In this regard, we show and extend this understanding to shorter time scales from larger time scales, as shown in the extended surveys for both SC paths of the VOYAGER mission, e.g. [2] [3].

Section 2 presents a few facts about the individual path of each SC through the HS and the criteria for selection of intervals in this survey. Section 3 shows the examples supporting the interpretation that, in all the scales we have surveyed, there is an MHD behavior of the SW in the HS dominated by compressional features. Conclusions and the discussion are given in Section 4.

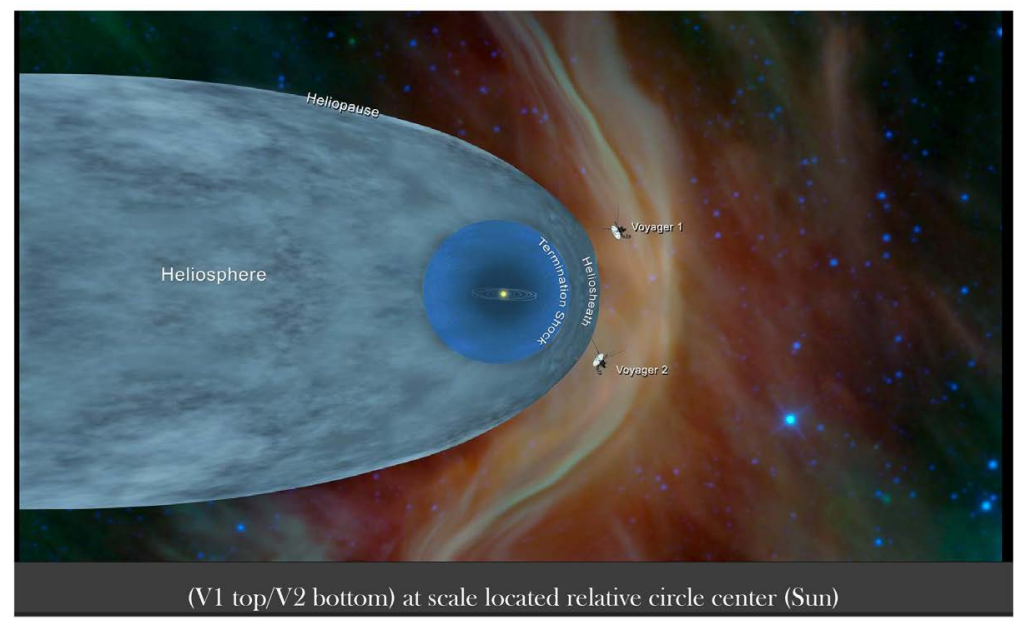

Figure 1. Artistic view of the sun in its interstellar neighborhood. 


\section{Journey and Trajectory of the SC in the HS/the Intervals Selection}

Notice that Voyager 1 (V1) moved north in the standard definition we use for the celestial sphere centered in the Sun, whose trajectory can be approximated as inertial. In this way simplifying the analyses for properties of the magnetic fields. See Figure 1.

The following web site https://ourplnt.com/space-probes-leaving-solar-system/\#axzz6btZESSf2 shows our Figure 1, an artistic representation of one of the few currently imagined visualizations of the Sun, at the center of a circle that represents the TS. Further, in Figure 1 the solar system is placed into context with its surroundings, including the very local inter-stellar medium (VLISM). Figure 1 represents the Sun surrounded by its built up MHD environment, generated by the expansion of a magnetized ionized matter continuously emitted by an overheated solar corona [9], see also the literature in [9], which expands in all directions away from the star supersonically until it encounters the TS, represented by a circle enclosing the point in its center (Sun). Beyond the circle is the magnetized plasma (SW) that continues moving further away at lesser speed (sub-magnetosonic). This is the region of our interest in this work, the HS. Figure 1 further shows: $1^{\text {st }}$ The V1/V2 platform on top/bottom right, both SC platform locations relative to the Sun at scale, while platform drawings out of scale; $2^{\text {nd }}$ the external to the SW magnetized matter. This surrounding region of the magnetized matter constitutes the so-called molecular cloud, dominated by neutral matter [10] with a certain percentage ( $15 \%$ to $35 \%$ ) of plasma with a temperature of 15 to $30 \times 10^{3}$ $\mathrm{K}$, and constitutes our LISM.

Although it is tempting to take at face value some first-hand observations, nature tends to play tricks on us regarding straight forward extrapolations. For example, sometimes, when V2 crossed the SW TS at 83.7 AU in the Southern hemisphere at $10 \mathrm{AU}$ closer to the Sun than was found by V1 in the North, then a TS clearly asymmetric [11] would be at first a reasonable assumption. But with the passing of time ideas are reviewed. Consequently, as we continue exploring more Voyager data in our analyses we find that maybe the asymmetric TS is not so asymmetric after all. While we as yet we do not know for sure the asymmetry or not of the regions crossed by the Voyager SC, several current studies suggest a closer to spherical TS. Current day interpretations of the TS are, in general, more in line with the artistic representation given in Figure 1.

In this work, with V2, we performed an analysis for the same length of time as that for V1. This is not easy to justify now and today we know that our V2 survey in the HS needs to be extended for a more complete comparison between V1 and V2 in the HS.

The subject of a strong variability of the B-field in the HS along the trajectory of V2 has been mentioned already [12]. There they reported that HS plasma and magnetic field were highly variable on scales as small as ten minutes. But they only mention that using one illustration. 
The top panel, in Figure 2(A), illustrates in blue the raw counts of the OU sensor along X PL, and in green same for the IN sensor, the red line constitutes the reference zero level for the OU, turquoise is the reference level for IN. The middle panel same for the OU and IN sensors along $Y$ PL direction. Bottom panel similar to the other two plots but for the $Z \mathrm{PL}$ direction, which quite precisely is along the orientation radial from the Sun for OU and IN.

The key features shown in Figure 2(A) are: 1) raw data for both magnetometers measured in counts, see e.g. [13], 2) off-sets from MAGROLs, 3) same time-evolution for sensors oriented similarly, indicating that medium features are well established, 4) lesser structures along radial from the Sun (Z PL direction), indicative of intensity mostly perpendicular to it, a constraint consistent by the Maxwell vector property $\operatorname{div}(\mathbf{B})=0$. We use this hypothesis in our study. For further constraints in the intensity of the $\mathbf{B}$-field along the MAGROL rotation direction [14].

Figure 2(B) shows, in the magnitude of the estimated magnetic field density observations for VI, features in the HS that qualitatively give a clear suggestion of its fundamental MHD compressible nature. This figure is taken from the interval of time in 2011 which extends for $1 / 3$ of the year prior to the arrival of V1 to the LISM (Its very local region to the HS).

After achieving the calibrated off-sets shown in Figure 2(A), Figure 2(B) gives the $\mathbf{B}$-field magnitude in [nT] from the OU instrument, for approximately $1 / 3$ of 2011 .

Figure 2(C) shows the overall view captured by V1 of the HS analyzing day-ave(-rage) of B-field within a different Coordinate System (RTN) as a function of time. This gives us an overall learning along the path of V1 from before to the TS (year 2004) to the HP in year 2012, near doy 220. See e.g. Stone announcement [15], Dec 2012. Marked in the left, with a vertical red-line is the time of the crossing of the TS. Top panel (A) shows with a shading under a solid black line the intensity of the magnetic field as a function of the time. The panel includes a red-line giving the energetic particles experiment estimate of the SW bulk speed as a function of time. Also comments on the regions along V1 path, in Figure 2(C), are indicated (black lettering). Middle panel (B) shows the dependence of the azimuth $\mathbf{B}$-field angle $(\lambda)$ on time. The panel includes lettering indicating interval corresponding to the Helio-sheet crossing for long lasting intervals. Bottom panel (C) shows the dependence on time of the latitude B-field angle $(\delta)$.

The whole interval of V1 in the HS is presented in Figure 2(C), further indicated in Figure 2(C) are the dependence of the B-field vector on time through its magnitude, top panel and in the middle and bottom panels its angular orientation. We keep present Figure 2(C) when discussing the difference in time interval of our study between V1 and V2, field variability, mean intensity, etc. Notice Figure 2(C)'s indicated evolution of plasma along the path of V1. The plasma velocity, indicated in red, is in the top panel of Figure 2(C). It is inferred 

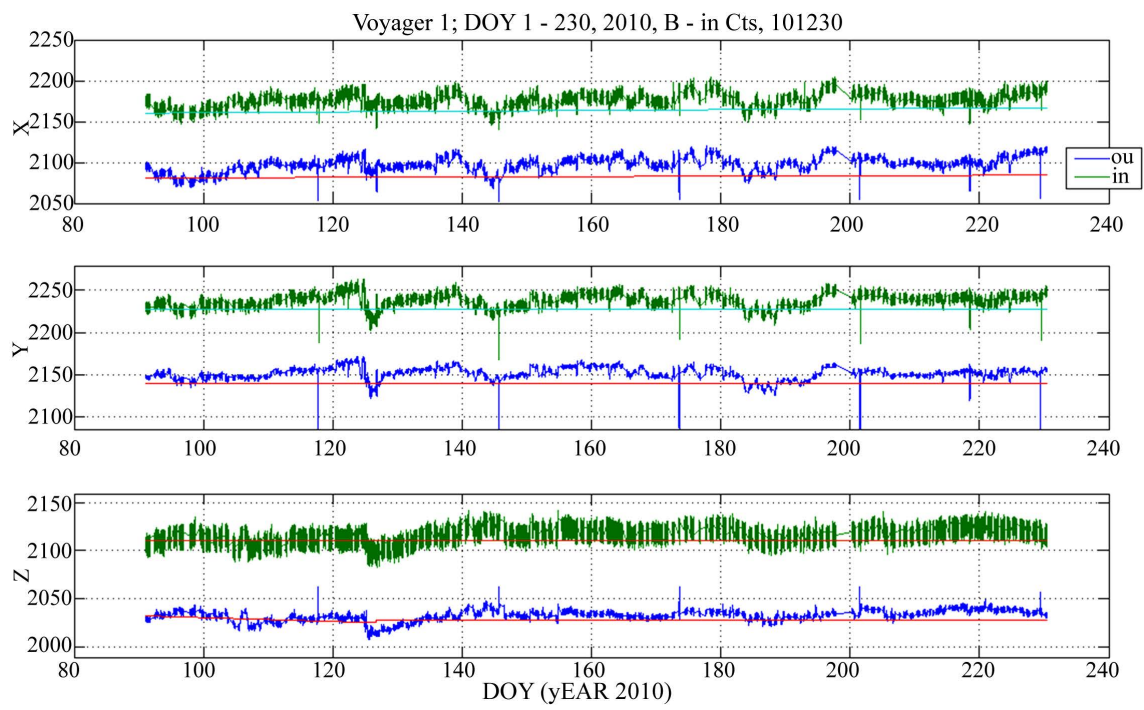

(A)

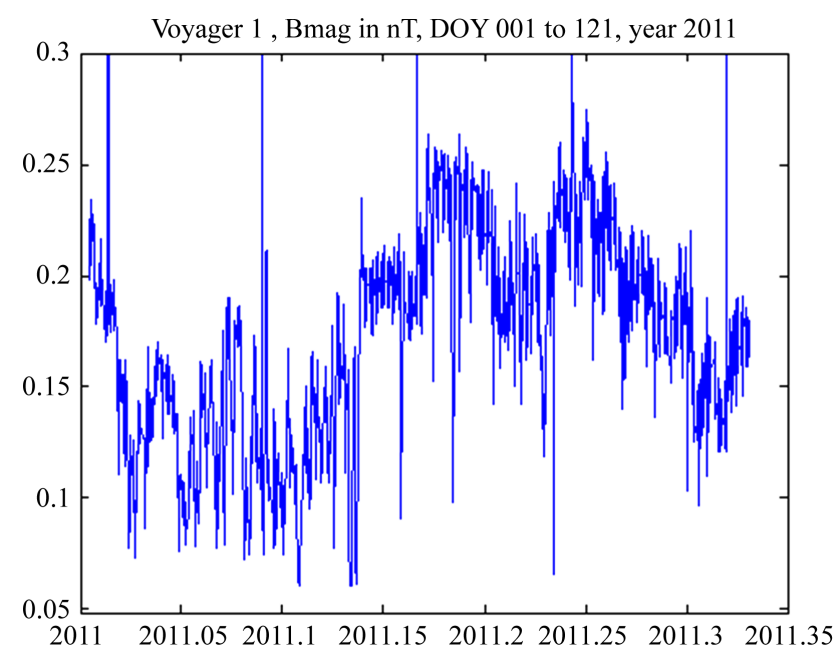

(B)

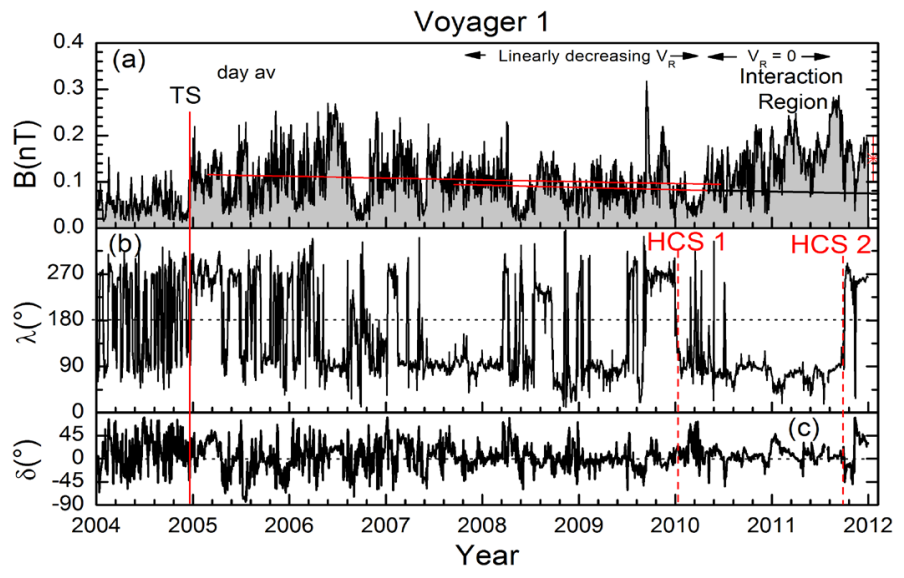

(C)

Figure 2. (A) Observations by V1 of the year 2011, 48 s ave B-field components; (B) Magnetic field magnitude observed by V1 in the $1^{\text {st }}$ third of year 2011; (C) Time interval spent by V1 in the sheath after solar wind (SW) termination shock (TS). 
indirectly from low energy accelerated particles. This is because of an early failure of the plasma instrument when V1 was in the heliosphere.

As already mentioned before the MAGROL, now with Figure 3 we proceed to show the manifestations in the data of a MAGROL when the SC performs ten turns (rolls) around an axis which passes through the center of the high-gain antenna of V1, which has telemetry with the deep-space antennas of the north hemisphere at Earth. The interval in Figure 3 presents one example of the MAGROL tools to generate the calibrated magnetic field provided to the science community at large. In this particular case, the interval shown extends in year 2006 from doy 17, 12:00 to 17:20 hh: mm (hours: minutes) universal time (UT). The key implications of this calibration tool are given in the Appendix. For more detailed analyses see e.g. [16].

The sinusoidal curve in each panel of Figure 3 represents the view of the B-field vector of the medium captured from each orthogonal $X$ PL and $Y$ PL axis, where we track the $\mathbf{B}$-field orientation perpendicular to the radial direction from the Sun during an early MAGROL from year 2006. The components along each one of both axes show a relative phase of $90^{\circ}$, see Appendix. The B-field along $Z \mathrm{PL}$ is/remains undetermined using this technique.

If the amplitude of the sinusoidal view in both panels of Figure 3 does not change over the interval of the ten rotations, $5 \mathrm{hr} 20 \mathrm{~min}$, the determined intensity of the $\left|\mathbf{B}_{\text {perp }}\right|$ (field $\mathrm{F}$ to radial from Sun) is unchanged. For more details on an estimate of the intensity of the B-field along $Z$ PL see e.g. [14].

Shown in Figure 3 are in the PL coordinate system components of the V1 B-field perpendicular to the radial from the Earth direction along $X$ and $Y$ PL (top and bottom panels respectively) as a function of the time but counted in the number of $48 \mathrm{~s}$ B-field average (ave) chronologically recorded vectors from the start of the year 2006, and corresponding to near 12:00 to 17:30 UT on the $17^{\text {th }}$ day of year (doy). The vertical axes indicate the values of the B-field in counts as a function of time. The center of the sinusoidal in top and bottom panel indicates the location of the zero-intensity of the field, see Appendix.
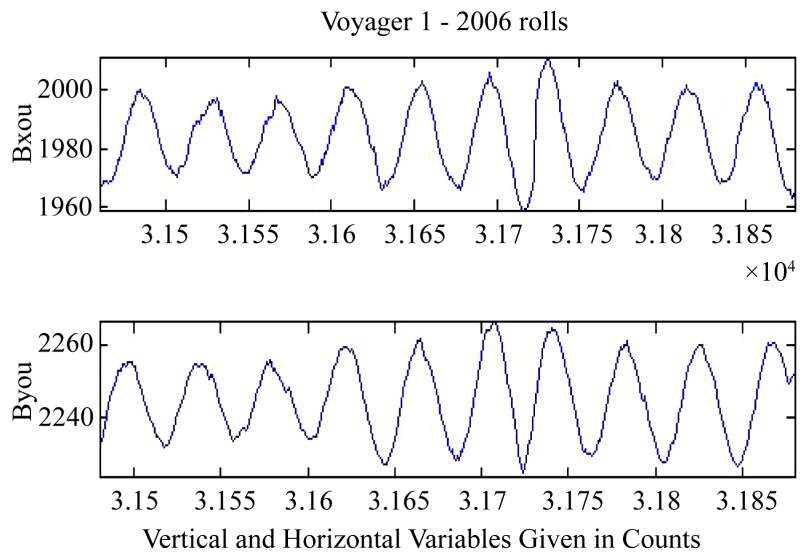

Figure 3. Example of the B-field during the rolling of the SC V1 around the $Z$ PL direction. 
The better, more precise measurement/knowledge of the B-field density occurs precisely at the times when we Roll (MAGROL) the SC in the above described way. These are our intervals selected for their comparison of the behavior of the B-field density along the path of V1 and V2.

Using the MAGCAL tool permits us to reduce the uncertainties in the determination of the B-field along $Z \mathrm{PL}$, the axis of rotation when performing the MAGROLs. The currently used technique is described in [14], and is the subject of further investigation. On the OU magnetometer in Voyager 2, its orthogonal orientation, due to their displacement in orientation, still constitutes a right handed orthogonal coordinate system, but displaced with respect to the $Y$ and $Z$ sensors along $Y$ and $Z$ PL, see e.g. [16].

Notice that during the MAGROL, and while the SC rotates with a period of about 32 minutes Figure 3 indicates the small and slow change as a function of time in the amplitude of the sinusoidal drawn by the measured intensity of the direction of the medium B-field density. This intensity evaluated in the form of amplitude in the plot shown in Figure 3 constitutes its component perpendicular to $Z$ PL. Figure 3's shown changes in amplitude indicate a possible manifestation of a compressional feature of the HS observed in-situ, the focus of the survey of the work.

While in most cases the feeblest of all components in the measured B-field density is the one radial from the Sun, $Z \mathrm{PL}$, sometimes that may not be the case.

However, it may be interesting to observe and, even more so to understand when occasionally that is the opposite, and that is precisely what occurred on day of year (DOY) 256, year 2008. At that time, as illustrated in Figure 4 for V2, the intensity of the $\mathbf{B}$-field mostly in the $Z \mathrm{PL}$ orientation. (For this case showing $\mathrm{Bz}$ PL $>\mathrm{B}_{\text {perp }}$ we are indebted to a private communication by Alan Cummings, 2020, of the Cosmic Particles Instrument in the Voyager mission.)

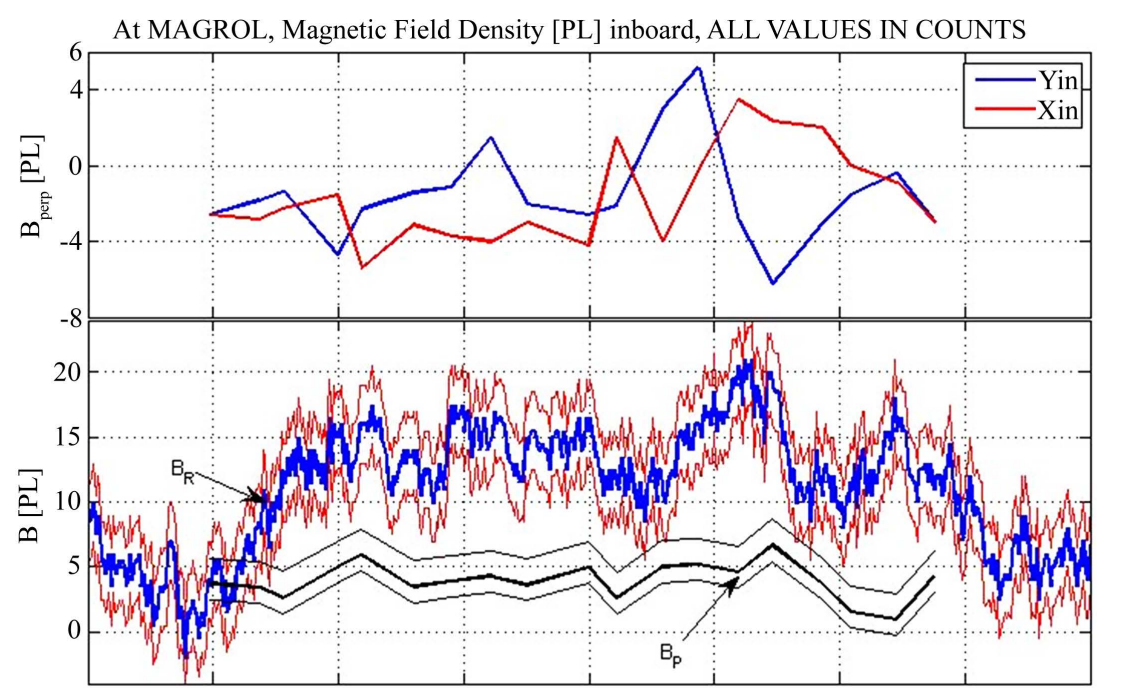

2008.6992008 .69912008 .69922008 .69932008 .69942008 .69952008 .69962008 .69972008 .6998

Figure 4. Unusual variation of the $X, Y$ and $Z$ PL components of the B-field during a V2 MAGROL. 
Figure 4 shows with its horizontal line the time dependence, while with its vertical axes intensity of the B-field in counts. B-field measured along the V2 path at the time of the MAGROL of doy 256-7, year 2008 are presented in the Top panel. The Bottom panel shows a stronger B-field in the radial direction from the Sun together with the intensity in its perpendicular direction. Further the bottom panel presents measurements and error of $[1+1 / 2] \sigma$ uncertainty.

\section{Examples}

Like in Figure 3 the following surveyed intervals are for maximum quality at/near the occasions when we do the in-situ MAGROL calibration of the magnetic field of the medium, see more in the Appendix. In this way we are able to provide analyses during intervals of time when there are the best and most precise magnetic field directions and intensities available.

\subsection{At 2 Years of the TS Crossing}

In Section 2, top panel of Figure 3 the conditions at V1 are shown; only plotted is the outboard component of $X \mathrm{PL}$ when the SC is rotating. and Figure 5 shows in its top panel the $X$ PL sensors of both IN and OU magnetometers in V2 at MAGROLs. The dramatic change in intensity of the B-field is self-evident in Figure 5. Here, the bottom panel shows the residual difference, indicative of the accuracy of the measurement. Notice that the uncertainty at each B-field vector measurement in the $X$ PL direction is between 2 and 3 Cts, which gives an uncertainty of $\pm 0.01 \mathrm{nT}$ for this component of the medium's B-field.

For the doy 78 - 79, year 2009, Figure 5 shows in its top panel for V2, the B-field $48 \mathrm{~s}$ ave as a function of time collected by both SC magnetometers along the $X$ PL direction measured in Cts for an interval containing a MAGROL.

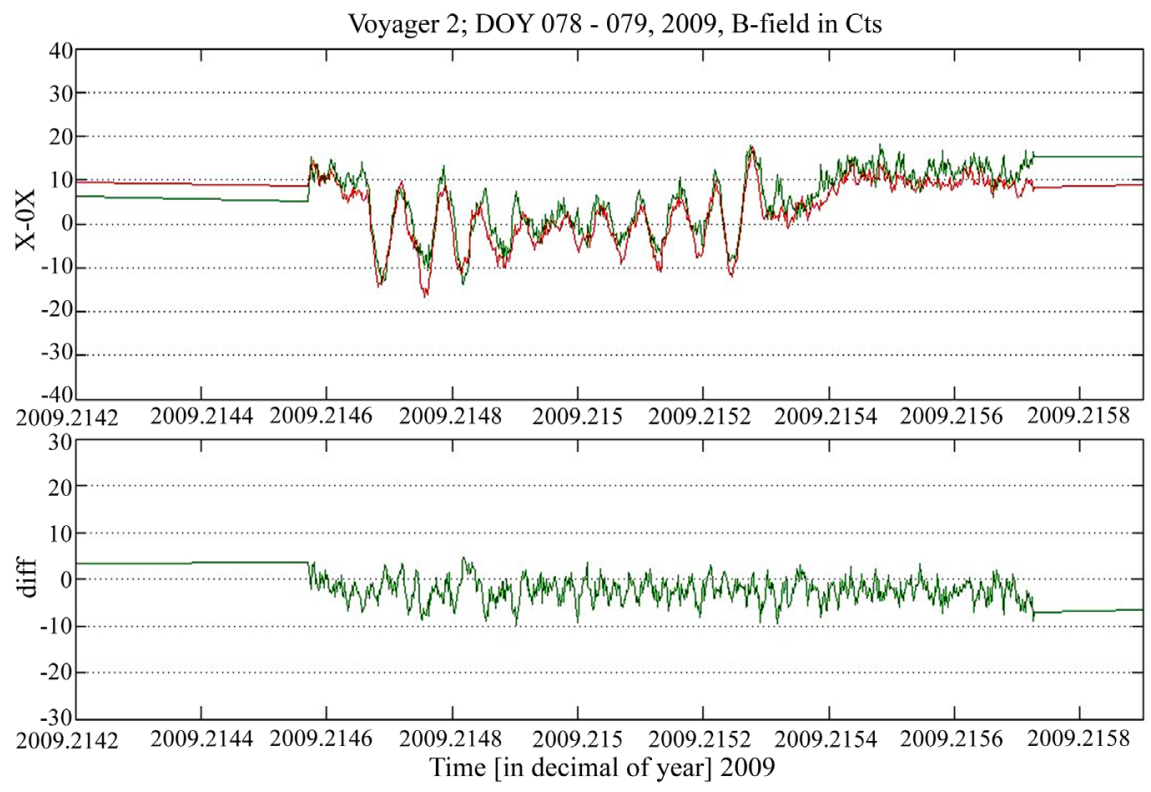

Figure 5. MAGROL on doy 78 - 79, V2 SC. 
Bottom panel gives the dependence with time of the difference between the calibrated observations as a function of time, same vertical scale than top panel, all in Cts.

\subsection{At 4 Years of the TS Crossing}

For V1, for the time interval indicated in Figure 6(A), shown is the B-field observed by both magnetometers measured by the $X$ PL sensors, like in top panel of Figure 5.

Figure 6(A) and Figure 6(B) show first V1 and second V2 respectively at MAGROLs . Figure 6 presents the agreement in the measurements of both magnetometers for MAGROLs on DOY 253 - 258, year 2008 for V1 and on DOY 258 - 261, year 2011 for V2. These plots show again the measurements from the sensors along $X \mathrm{PL}$, and the MAGROL determined zero intensity is the reference in counts units. Once more but less dramatically Figure 6(B) shows more magnitude fluctuation along the V2 path than the V1 path.

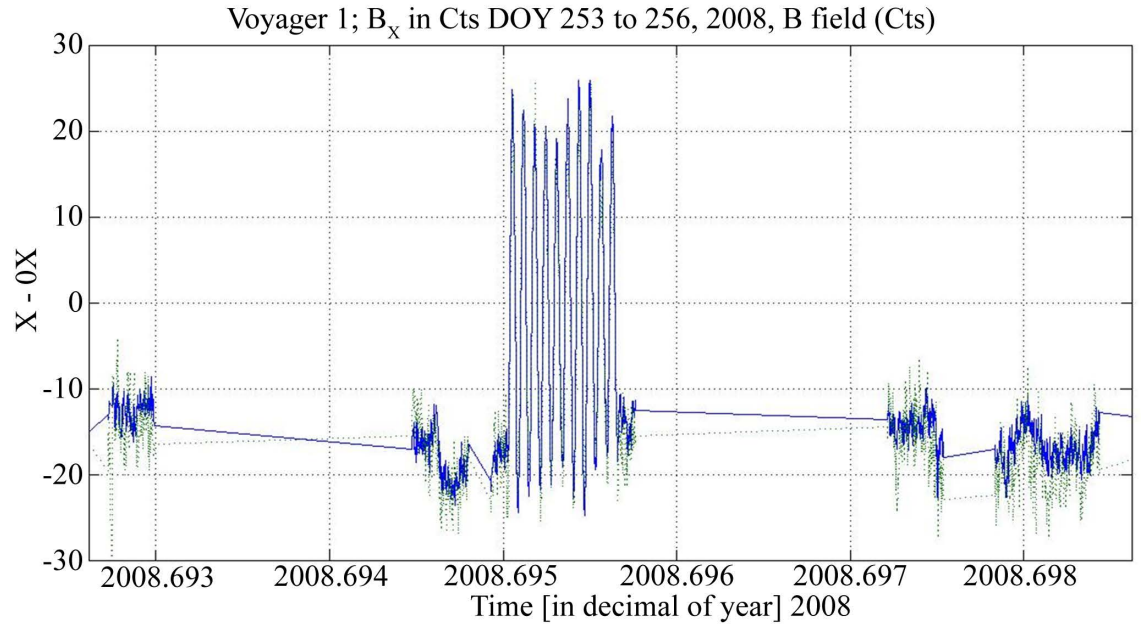

(A)

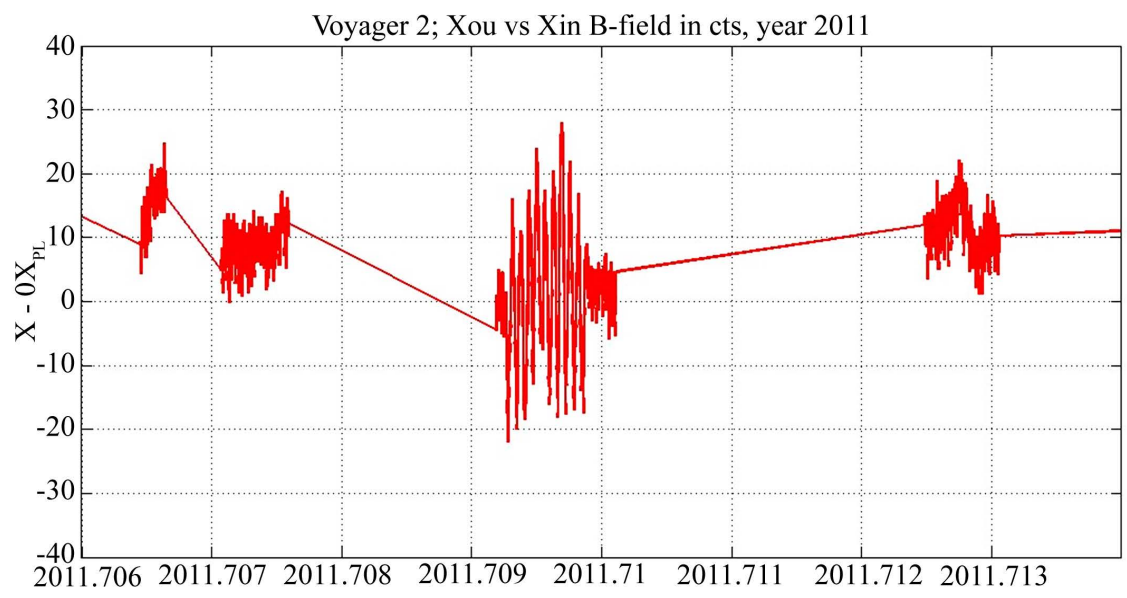

(B)

Figure 6. (A): V1 MAGROL for the time interval doy 256 - 257, year 2008; (B): For V2, ibid (A), for the time interval doy 259, year 2011. 


\subsection{At 5 Years of the TS Crossing}

Figure 7(A) and Figure 7(B) present observations at MAGROLs after 5 years of crossing the TS.

Figure $7(B)$ is similar to Figure 6. Figure $7(A)$ shows in its top panel the signal by both magnetometers along $X$ PL while in its bottom panel it shows the difference between the $X$ PL sensor measurements, which give an estimate of counts uncertainty. In this case this appears to be about \pm 4 cts and corresponds to an uncertainty of $0.02 \mathrm{nT}$ (at $0.005 \mathrm{nT} / \mathrm{cts}$ ), which is similar but larger than the uncertainty shown by the bottom panel in Figure 5.

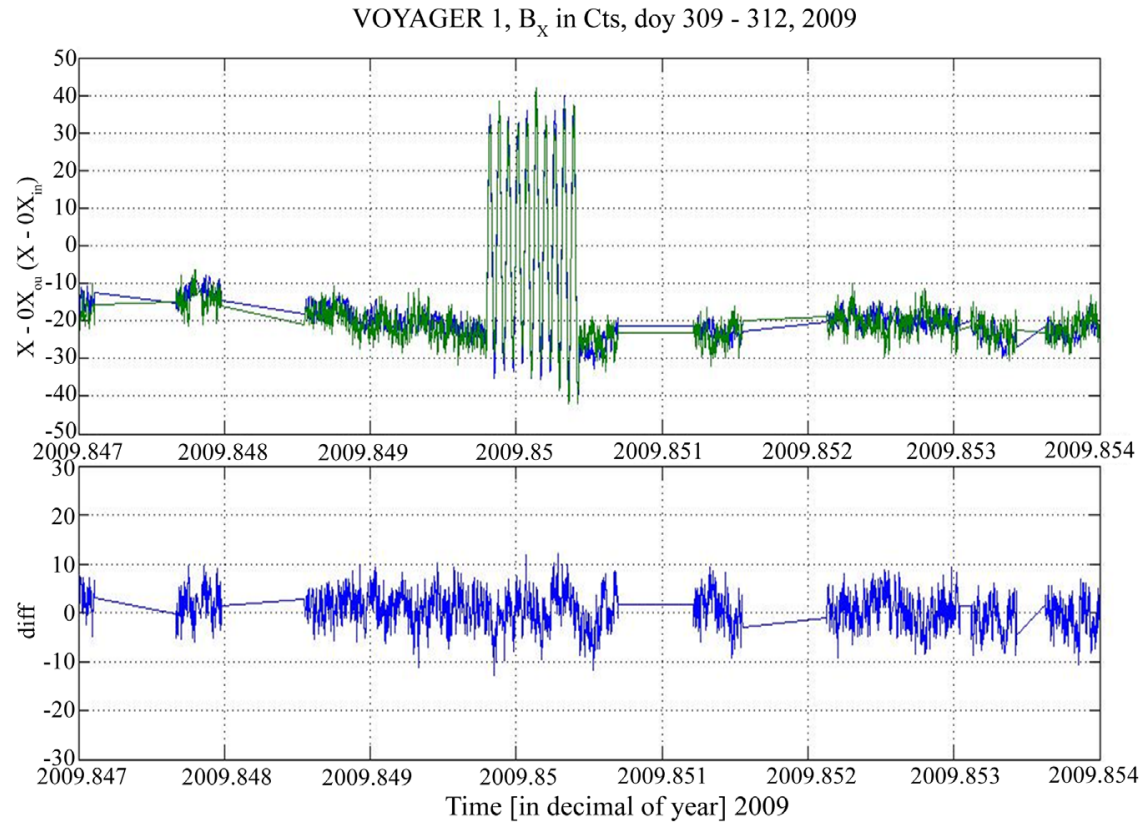

(A)

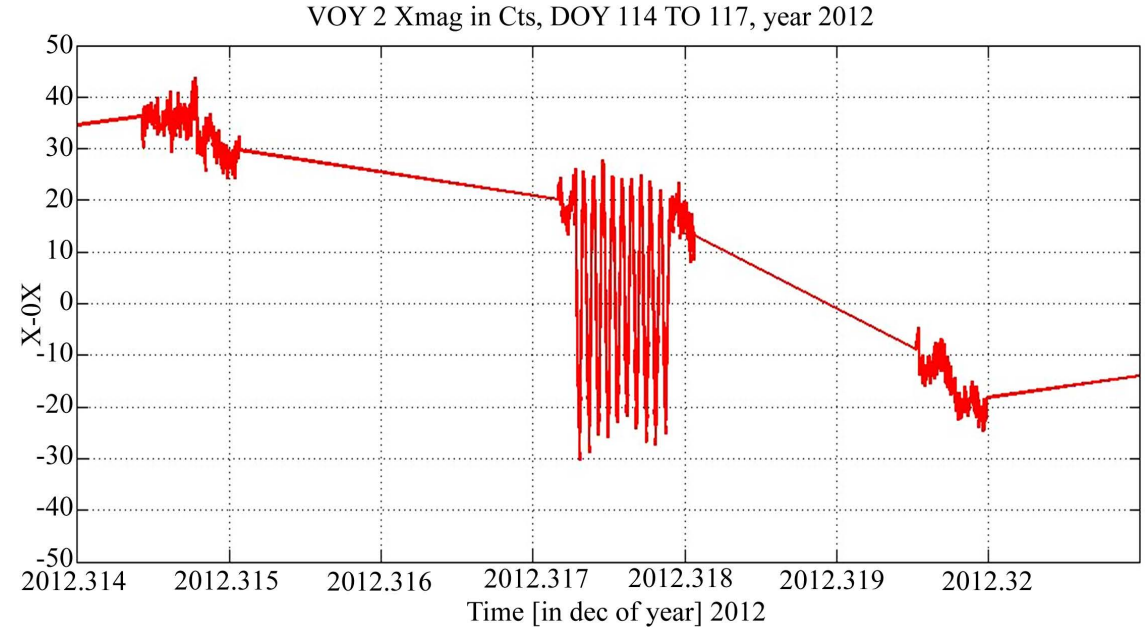

(B)

Figure 7. (A): ibid Figure 5 for observations with V1 for doy 309 - 312, year 2009, including MAGROL; (B): ibid Figure 6(B) for observations with V2 for doy 258 - 261, year 2011, includes MAGROL. 


\subsection{At 6 Years}

Figure 8(A) is like the previous Figure 7(A), but describes observations made a year later. It covers the time interval extending from DOY 126 to DOY 128, 2011. In much the same way, Figure 8(B) describes observations made a year later from those made for Figure 7(B), DOY 345 to DOY 348, 2013. Both figures show the agreement in the observations between IN and OU magnetometers.

\subsection{At 7 Years}

Figure 9(A) (top) and Figure 9(B) (bottom) show the first V1 on DOY 264, 2011 and the second V2 on DOY 309, year 2014 respectively at MAGROLs.

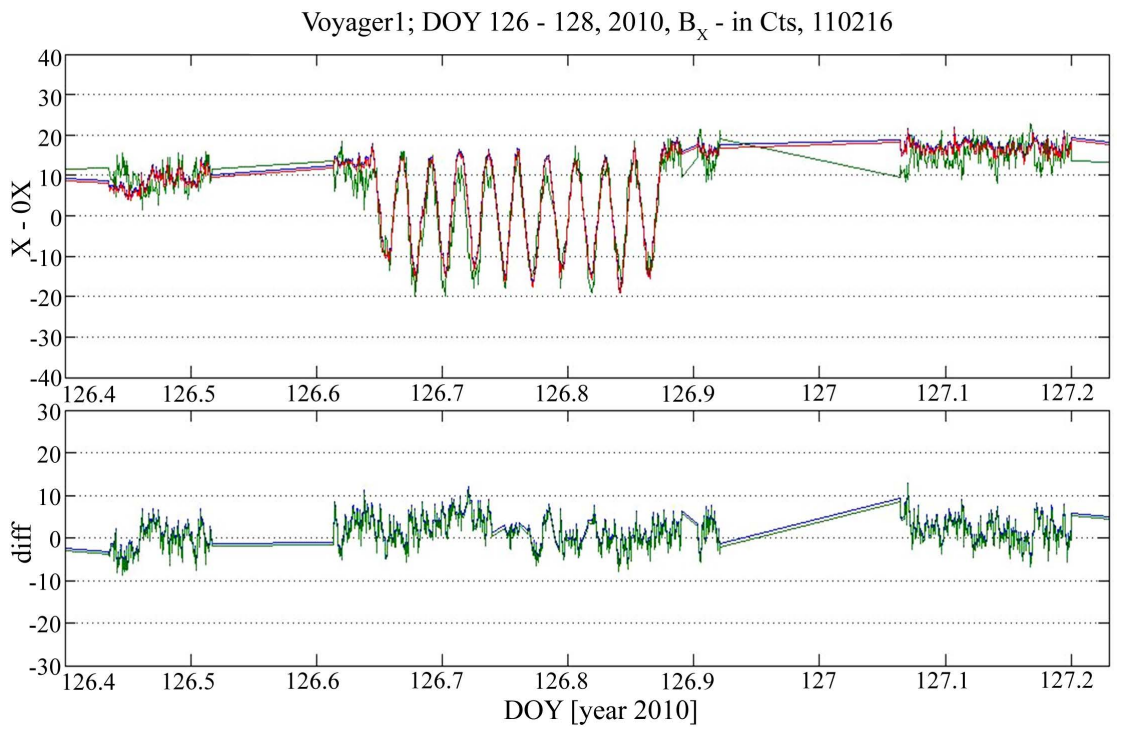

(A)

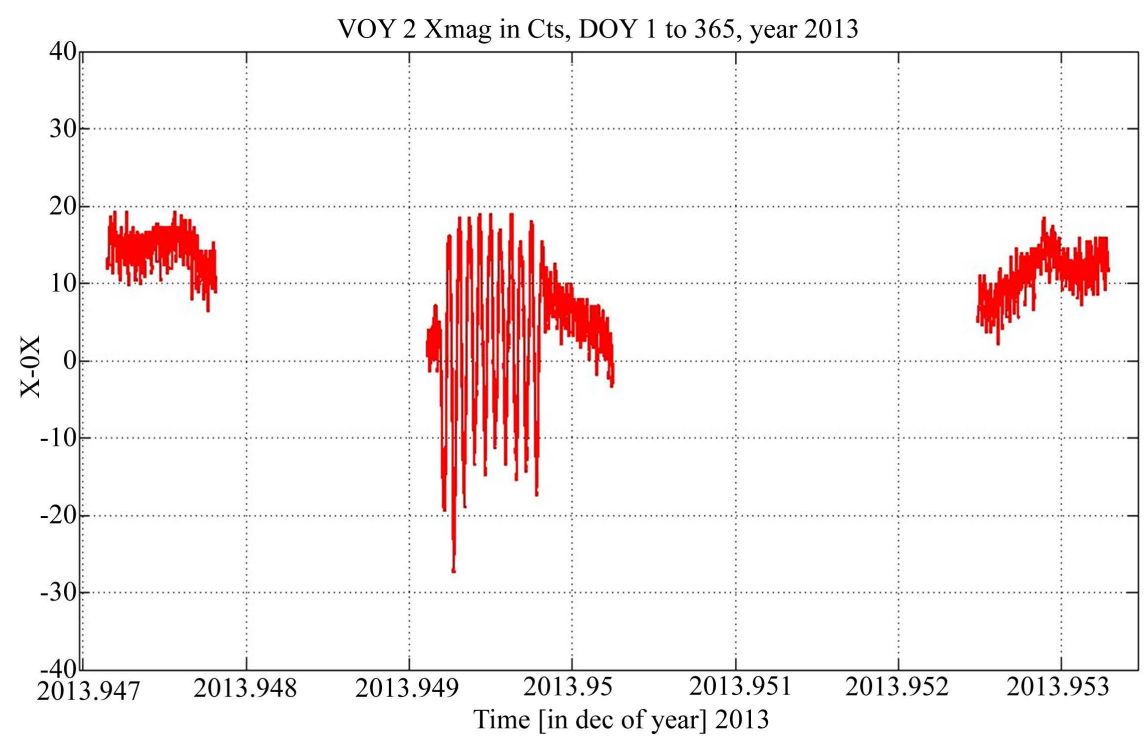

(B)

Figure 8. (A) and (B) show top V1 and bottom V2 respectively in different intervals at MAGROLs. 


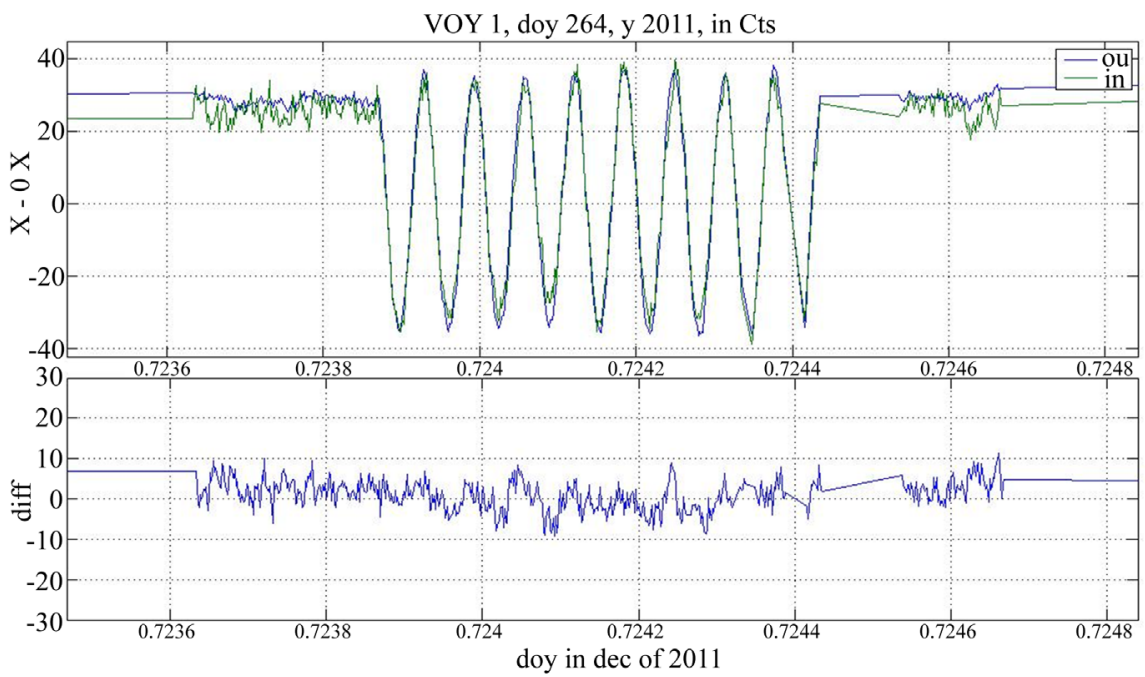

(A)

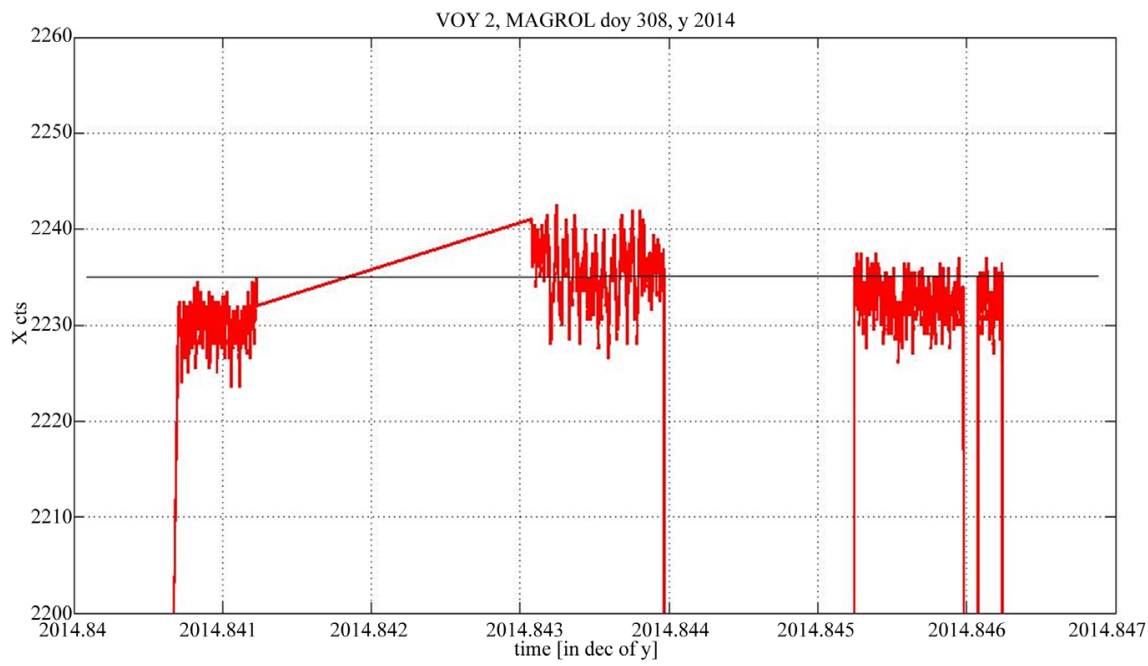

(B)

Figure 9. (A): ibid Figure 6(A) forV1 observations on doy 264, y. 2011; (B): ibid Figure 6(B) for doy 306 - 309, y. 2014, containing MAGROL interval.

Again Figure 9(A) presents on its top panel B measurements of the medium along X PL from both magnetometers. Interestingly its bottom panel indicates that in this case the uncertainty is closer to $\pm 0.01 \mathrm{nT}$ (about 2 cts at this moment in time). Figure 9(B) is similar to Figure 3 in the sense that it gives the MAGROL directly in observations scale as performed. However, as identified by this MAGROL, the horizontal full black line indicates the reference zero value of $B_{x}$ PL.

\subsection{V1 and V2 at HS, before/at Their Crossings Mid-2012 and near End 2018 Respectively}

After 8 years V1 arrived at the HP, moving at greater speed away from the Sun than V2. It took much longer to V2 to arrive at the HP. However, as we will check the arrival took place closer to the Sun. 
Considering the good approximate calibration " $0.005 \mathrm{nT} / \mathrm{Ct}$ " for each sensor of the magnetometer. Figure 10(A) and Figure 10(B) show that since before DOY 212 to after DOY 264, and already into the LISM the intensity of the B-field is mostly along $X$ PL. And this B-field orientation along the V1 path extends until our latest adjustment of the V1 zeroes, DOY 180, 2020 (present day).

Shown, in Figure 10(A), is one year of the $X$ PL component of the B-field $48 \mathrm{~s}$ ave in counts, from the start of year 2012 as function of time in decimal of year. Data of IN and OU sensors are color coded in inset.

In Figure 10(B) is shown approximately $1 / 3$ of one year of the magnitude of $48 \mathrm{~s}$ ave of the B-field in $\mathrm{nT}$ units containing the estimated location of the passage of V1 from the HS into the VLISM. Arrows indicate the connection of this interval with the one shown in Figure 10(A). Figure 10(B) expands the region from Figure 10(A) which identifies the transition from the HS into the LISM about DOY 234, year 2012. Notice the complex details of the passage by V1 recorded by the in-situ measurements of both V1 magnetometers Here, V1 B-field time series shows a complex passage illustrated also in Cts in Figure 10(A) through the component $X \mathrm{PL}$.

After 11 years V2, moving at less speed in the HS than V1, crossed the HP nearer to the Sun than V1 did (about 16 AU nearer to the Sun, on doy 309, 2018). V1 passage seen in Figure 11, is also complex.
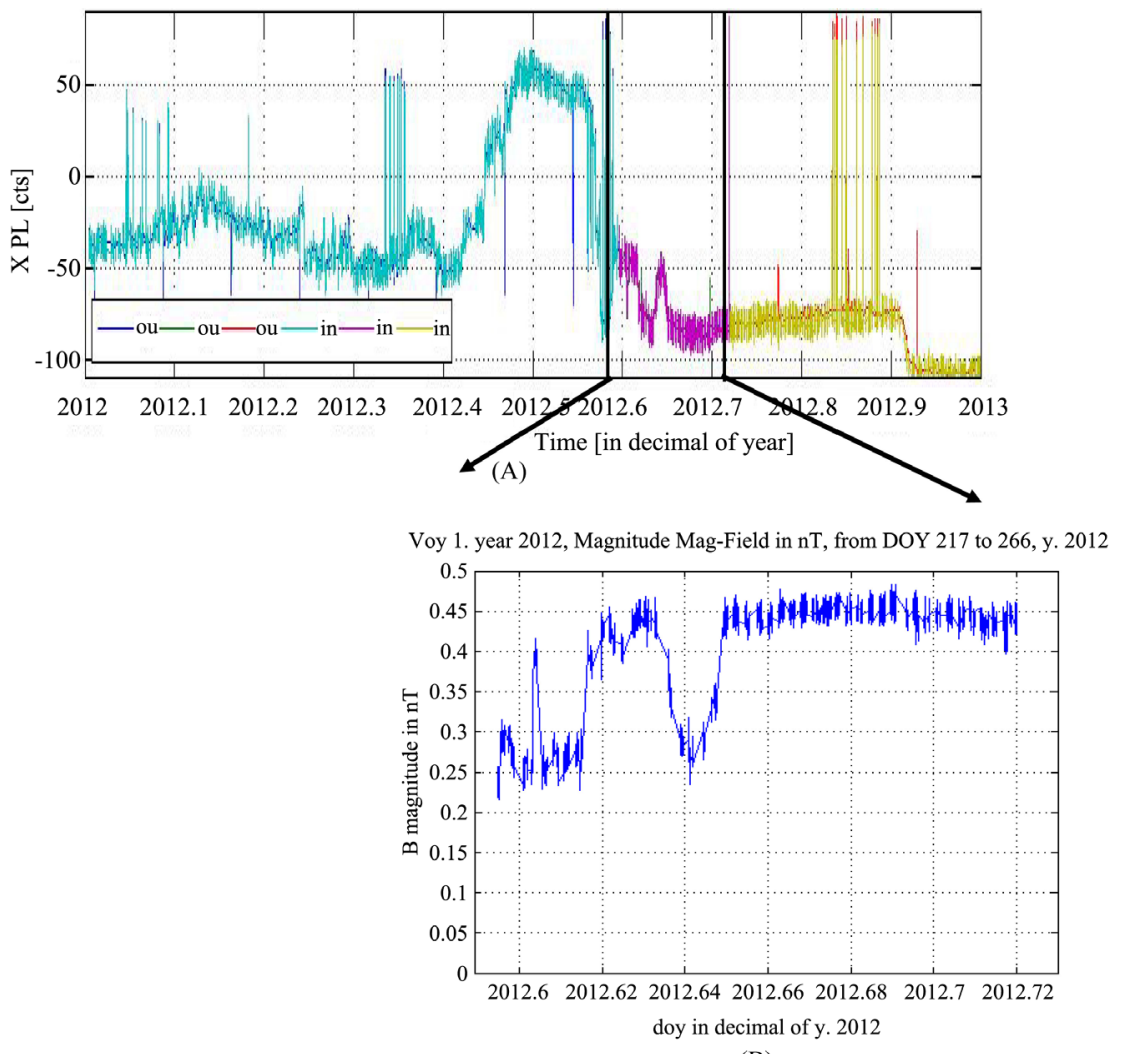

(B)

Figure 10. (A): The evolution of $X \mathrm{PL}$ along V1 path in the year it arrives to the HP; (B): From the HS, through the HP, and into the VLISM. 


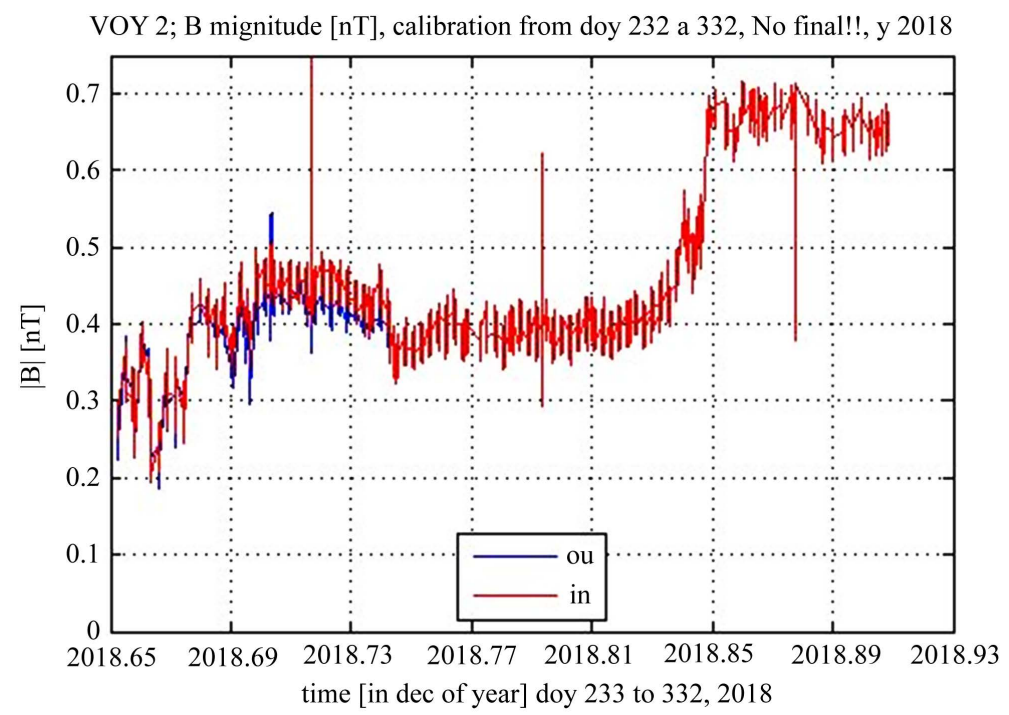

Figure 11. Ibid to Figure 10(B) but showing the $48 \mathrm{~s}$ ave magnitude of B along path of V2 crossing from the HS into the LISM.

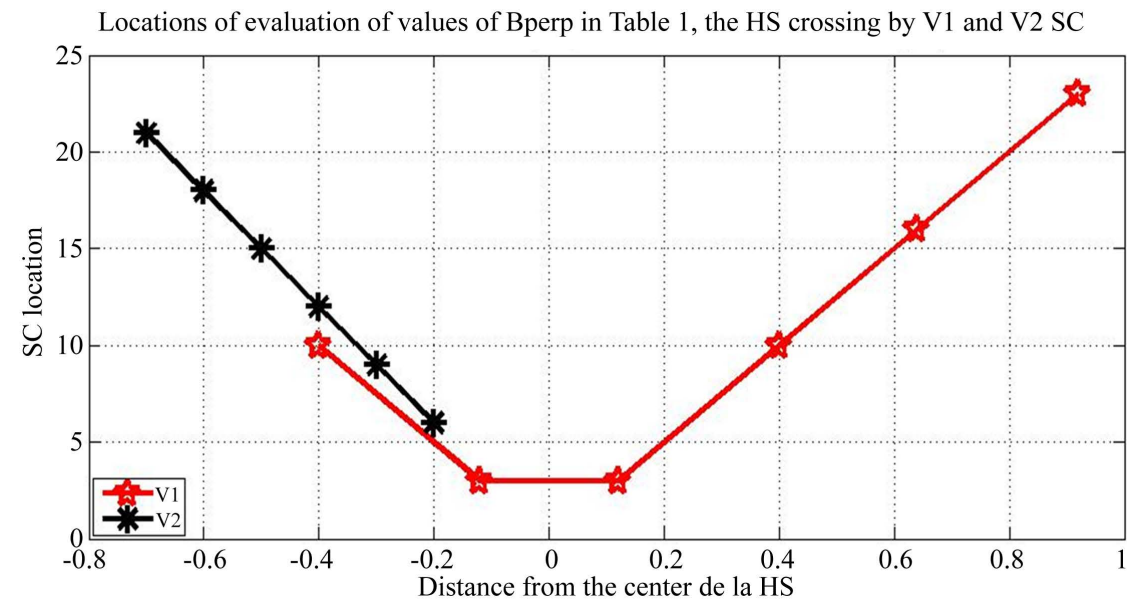

(A)

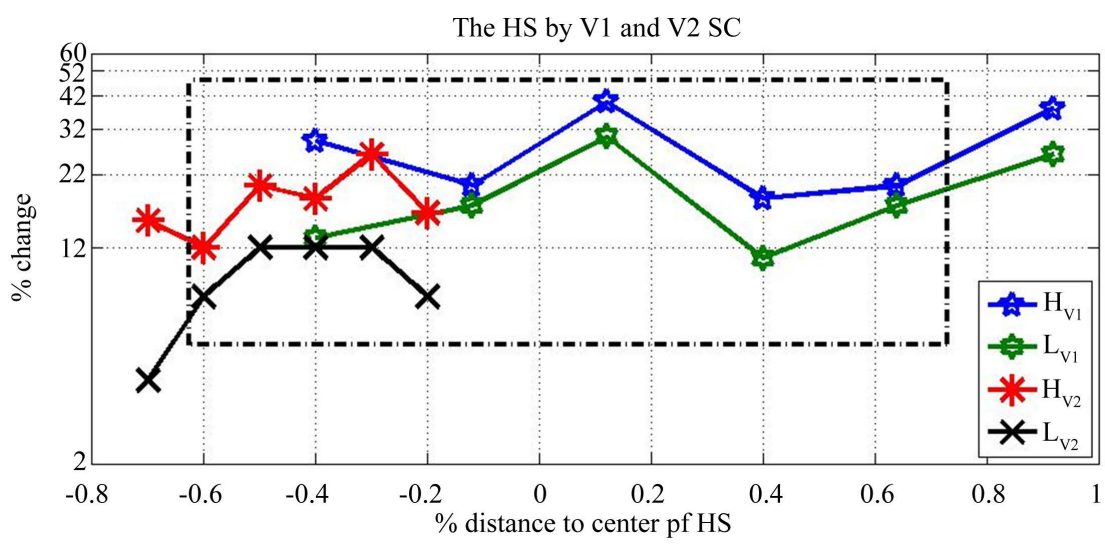

(B)

Figure 12. (A) Vertical axis gives in AU the SC V1 and V2 locations at observation time relative to assumed center of $\mathrm{HS}$ as described in the body of the text; (B) Highs (H) and Lows (L) at V1 and V2 as a function of distance from center of HS. 
Table 1. Quantitative values for the total of 12 selected intervals at MAGROL.

\begin{tabular}{ccccccccc}
\hline From TS & $\begin{array}{c}B_{\text {perp }}(\mathrm{V} 1) \\
(2006-2011)\end{array}$ & $\begin{array}{c}\mathrm{B}_{\text {perp }} \\
\text { at V1 }\end{array}$ & \begin{tabular}{r}
$r(\mathrm{~V} 1)$ \\
\cline { 2 - 8 }
\end{tabular} & $\begin{array}{c}\text { Largest } \\
\text { Smallest }\end{array}$ & $\begin{array}{c}B_{\text {perp }}(\mathrm{V} 2) \\
(2009-2014)\end{array}$ & $\begin{array}{c}\mathrm{B}_{\text {perp }} \\
\text { at V2 }\end{array}$ & $r(\mathrm{~V} 2)$ \\
\hline $2^{\text {nd }}$ year & 29 & 13 & 120 & 112 & 15 & 4 & 275 & 89 \\
$3^{\text {rd }}$ year & 20 & 17 & 18 & 119 & 12 & 8 & 50 & 92 \\
$4^{\text {th }}$ year & 40 & 30 & 40 & 125 & 20 & 12 & 65 & 95 \\
$5^{\text {th }}$ year & 18 & 11 & 64 & 132 & 18 & 12 & 50 & 98 \\
$6^{\text {th }}$ year & 20 & 14 & 43 & 138 & 26 & 12 & 117 & 101 \\
$7^{\text {th }}$ year & 38 & 26 & 46 & 145 & 16 & 8 & 100 & 104 \\
Mean & $27 \pm 4$ & $19 \pm 3$ & $42 \pm 16$ & & $18 \pm 2$ & $9 \pm 1$ & $110 \pm 36$ & \\
\hline
\end{tabular}

\section{Discussion and Conclusions}

Here we notice that half the crossing of the HS by V1 takes place at $122 \mathrm{au}$, while for V2 about half of the crossing of the HS takes place at $\sim 110$ au. Then we notice from our quantitative evaluation of Table 1, that avoiding compression effects possible by the TS and the VLISM on the HS, requires a comparison of years 3 to 5 , after the crossing of the TS (V1), with all cases after the $3^{\text {rd }}$ year of the crossing of the TS (V2) included. For this subset of our study we identify comparable average B-field in both corresponding HS regions in the South and North hemisphere.

The more compressional nature of the behavior of the HS along the trajectory of V2 than V1 is proven from the examples presented (see Table). However, more research is needed to achieve a more precise understanding of the degree of difference between the two paths along which the V1 and V2 platforms moved through the HS. It would be straightforward to achieve this purpose by expanding our case analysis to include all MAGROLs in the HS.

Nevertheless, we proceed with our available and precise measurements by analyzing and focusing on what we understand is the HS region less affected by boundary conditions, i.e. the impact of the TS and the HP of the very (V-) LISM, i.e. the boundaries of the HS. See the following discussion of Figures 12. In addition we consider that the transition into the VLISM may provide information on the nature of features of the HS of a possibly global character, which we so far have not noticed.

In Figure 12, the ordering of the selected set of cases expressed as a function of their distance to the middle of the HS provides a better picture of the overall characteristics of the conditions observed. Here, we realize that our results show an overall consistency with the daily averages, already documented [2] for V1, see also Figure 2(C), as well as the recently accepted work [3] on V2. Dot-point-rectangle in Figure 12(B) encloses the cases of interest. In this way, with Figure 12(B) it is presented a quantification of the pressure variations during MAGROLs as a function of distance from the center of the HS as inter- 
preted in the body of the work, discussion and conclusions.

By the time this report is written, we know more about the time when V2 finished its path through the HS. It was in this way that V2 found itself, by end of year 2018, beyond the boundaries of the HS (see Figure 11). We have also learned that the HS, in mean value at the time of each SC passage, is observed to be no more than $0.02 \mathrm{nT}$ stronger along the $\mathrm{V} 1$ path north than along the $\mathrm{V} 2$ path south in an inertial coordinate system centered on the Sun. We further know that for the weaker B-field along the V2 path magnetic compression demands less magnetic work, see e.g. discussion on the MHD state [1]. This higher compressional feature along the V2 path is consistent with a weaker field intensity encountered in the HS by V2 than by V1.

This invited paper is based on a presentation at the 2017 American Geophysical Union Fall Meeting. The exploratory approach there, for simplicity to compare equal intervals of time in the HS for both SC, was adopted ahead of the arrival of V2 to the HP. In hindsight, by now after the arrival of V2 to the local inter-stellar medium (LISM) it is clear that a better understanding will be achieved in the comparison of both SC by studying in equal footing the amount of time of both SC in the HS. Stay tuned for future completion of a work on this project.

\section{Acknowledgements}

Work had partial support from NASA grant NNG15CR65C. To my son Santiago for a thorough, careful editing of the US-English used for this scientific document, and to A. Cummings, 2020 for information of MAGROL time-interval with unusual orientation of the magnetic field density. I am further indebted to Dr L. F. Burlaga for facilitating the use of his unpublished plot, Figure 2(C). I am deeply in debt with my late wife for her encouraging, sweet, long friendship, and to my late father Carlos David, for his love of a whole life.

\section{Conflicts of Interest}

The author declares no conflicts of interest regarding the publication of this paper.

\section{References}

[1] Jackson, D.J. (1971) Classical Electrodynamics traducción directa del inglés por G. Marsal Aleixandre. Bs As, Bs As, Argentina.

[2] Burlaga, L.F. and Ness, N.F. (2014) Voyager 1 Observations of the Interstellar Magnetic Field and the Transition from the Heliosheath. The Astrophysical Journal, 784, 146. https://doi.org/10.1088/0004-637X/784/2/146

[3] Burlaga, L.F., Berdichevsky, D.B., Jian, L.K., Koval, A., Ness, N.F., Park, J., Richardson, J.D. and Szabo, A. (2020) Magnetic Fields Observed by Voyager 2 in the Heliosheath. The Astrophysical Journal, 906, 119. https://doi.org/10.3847/1538-4357/abccd1

[4] Berdichevsky, D., Thejappa, G., Fitzenreiter, R., Lepping, R., Yamamoto, T., Ko- 
kubun, S., McEntire, R., Williams, D. and Lin, R.P. (1999) Widely Spaced Wave-Particle Observations during GEOTAIL and WIND Magnetic Conjunctions in the Earth's Ion Foreshock. Journal of Geophysical Research, 104, 463-482. https://doi.org/10.1029/1998JA900018

[5] Berdichevsky, D., Szabo, A., Lepping, R.P., Viñas, A.F. and Mariani, F. (2000) Interplanetary Fast Shocks and Associated Drivers Observed through the Twenty-Third Solar Minimum by WIND over Its First 2.5 Years. Journal of Geophysical Research, 106, 25133-25133. https://doi.org/10.1029/2001JA000074

[6] Burlaga, L.F. (1995) Interplanetary Magnetohydrodynamics. Oxford University Press, New York.

[7] Berdichevsky, D.B., Farrugia, C.J., Lepping, R.P., Richardson, I.G., Galvin, A.B., Schwenn, R., Reames, D.V., Ogilvie, K.W. and Kaiser, M.L. (2003) Solar-HeliosphericMagnetospheric Observations on 23 March-26 April 2001: Similarities to Observations in April 1979. AIP Conference Proceedings, 679, 758-762. https://doi.org/10.1063/1.1618703

[8] Farrugia, C.J. and Berdichevsky, D.B. (2003) Evolutionary Signatures in Complex Ejecta and Their Driven Shocks. Annales Geophysicae, 22, 3679-3698.

https://doi.org/10.5194/angeo-22-3679-2004

[9] Berdichevsky, D.B., Rodriguez-Gomez, J.M., Vieira, L.E. and Dal Lago, A. (2020) Thermodynamics Interpretation of Electron Density and Temperature Description in the Solar Corona.

[10] Rankin, J.S., McComas, D.J., Richardson, J.D. and Schwadron, N.A. (2019) Heliosheath Properties Measured from a Voyager 2 to Voyager 1 Transient. The Astrophysical Journal, 883, 101. https://doi.org/10.3847/1538-4357/ab3d9d

[11] Stone, E., Cunnings, A., McDonald, F.B., Heilila, B., et al. (2008) An Asymmetric Solar Wind Termination Shock. Nature, 454, 71-74.

[12] Richardson, J.D. and Burlaga, L.F. (2013) The Solar Wind in the Outer Heliosphere and Heliosheath. Space Science Reviews, 176, 217-235.

https://doi.org/10.1007/s11214-011-9825-5

[13] Behannon, K.W., Acuña, M.H., Burlaga, L.F., Lepping, R.P., Ness, N.F. and Neubauer, F.-M. (1977) Magnetic Field Experiment for Voyager 1 and Voyager 2. Space Science Reviews, 21, 235-257. https://doi.org/10.1007/BF00211541

[14] Berdichevsky, D.B. (2015) Voyager Mission, Detailed Processing of Weak Magnetic Fields; II-Update on the Cleaning of Voyager Magnetic Field Density B with MAGCALs. http://vgrmag.gsfc.nasa.gov/20151017BzPLestimates wMAGCAL.pdf

[15] Stone, E. (2012) Voyager 1 Spacecraft Detects Particles at Solar System's Edge. Space, from Clara Moskowitz Interview, Editorial Page of Science \& Astronomy, December.

[16] Berdichevsky, D.B. (2009) Voyager Mission, Detailed Processing of Weak Magnetic Fields; I-Constraints to the Uncertainties of the Calibrated Magnetic Field Signal in the Voyager Missions.

http://vgrmag.gsfc.nasa.gov/Berdichevsky-VOY sensor_opu090518.pdf

[17] Goldstein, H. (1951) Classical Mechanics. Addison-Wesley, Boston. 


\section{Appendix}

Since the launch of the Voyager missions V1 and V2, the routine is to use as a calibration tool the rolling of the SC (in a MAGROL) at approximately a rotation every 38 min producing for a fixed direction of the magnetic field the identification of the B-field displayed below in Figure A, in the direction orthogonal to the axis of rotation, direction $Z$ PL.

A careful analysis, present in our tool-box identifies for us that the intensity zero for the $X \mathrm{PL}$ direction is located at $X \mathrm{ou}=2036 \pm 1$ counts (Cts) and for the $Y$ PL direction at $Y$ ou $=2158 \pm 1$ Cts.

The following Appendix, Appendix Bis A, surveys the limitations of the present ability to evaluate the intensity of both orthogonal $X$ and $Y$ PL compo-

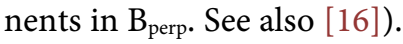

\section{Appendix Bis A.}

Here we simplify the discussion to the treatment of the IN (-board) coordinate system which is simply oriented along the PL coordinate system, We can afford to do that because the agreement at MAGROL between PL and OU (-board) coordinate system is well proved at the level of the evaluation of zeroes of the B-field at the present stage of analysis and we illustrated that in Figure A1. (This is so despite a mild distortion of a few counts by a spurious wave of arbitrary amplitude present in the OU sensor $X$ at the MAGROL.)

Classical Mechanics, Herbert Goldstein, 1951 text book [17]) permits us to use the transformation.

$$
\begin{aligned}
& X(t)=X_{F I X} \cos (\omega t+\phi)+Y_{F I X} \sin (\omega t+\phi) \\
& Y(t)=-X_{F I X} \cos (\omega t+\phi)+Y_{F I X} \sin (\omega t+\phi)
\end{aligned}
$$

See Figure 3, Figure A, and Figure A1.

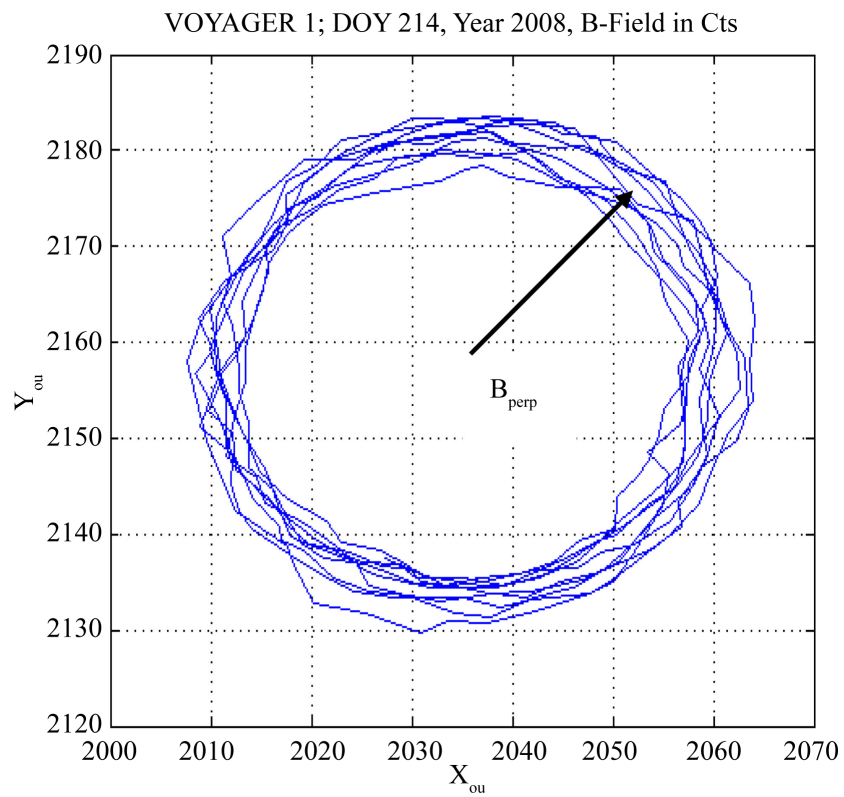

Figure A. Shown is for a V1 MAGROL, $Y(X)$ in PL coordinate, data from OU magnetometer. 


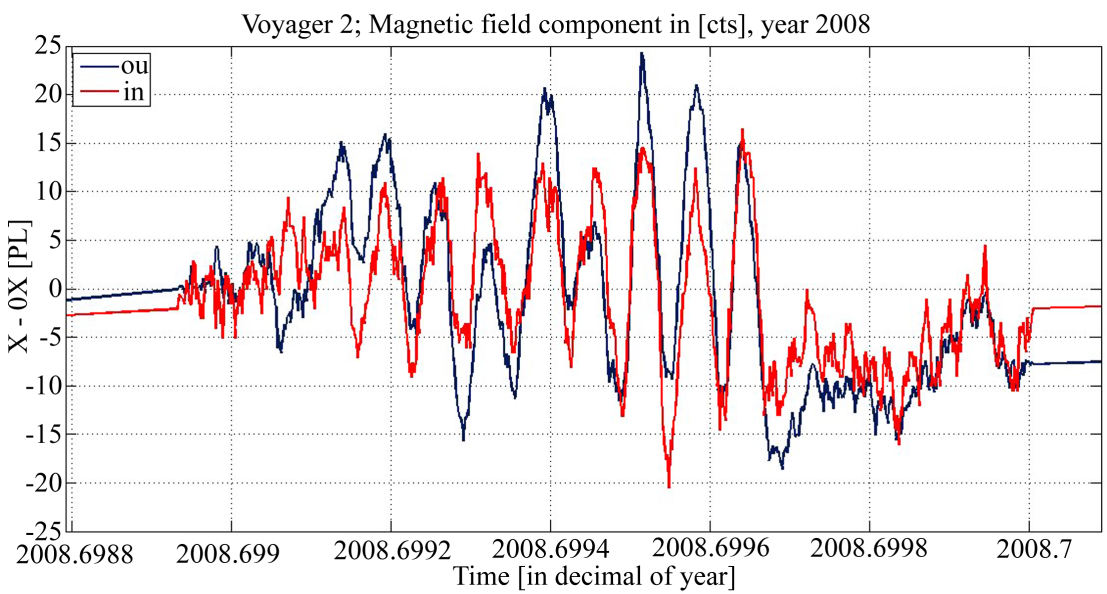

Figure A1. Plot of the intensity evaluated at MAGROL along the line of observation of the $X$ PL for the OU (blue line) and IN (red line) instruments, along the V2 path on DOY 256 , year 2008 .

Figure A2 illustrates the use of the fast fourier transform (FFT) to the interval of the MAGROL shown in Figure A1 from time interval 2008.6991-2008.6996, which is evaluated in decimal of year, that corresponds to about $28 \mathrm{~min}$. period. (The user of this FFT must be aware among other facts that the method provides significant/meaningful values only up to half of the plotted interval, and in a simple case like this with a resonating frequency " $P$ " like in this case one of the values is spurious, as it is shown here). The valid obtained value is the frequency $f=1 \times 10^{-5}$ year $^{-1}$. The representation is with the phase $\phi=9 \pi / 16$. Hence, we proceed to plot generically the $X(t)$ value together with $Y(t)$ with the phase $\phi$ (listed in the sentence above).

And we apply this value to a simple test of constant fields for the period of the MAGROL interval of ten rotations, see Figure A3. There, $X(t), Y(t), X_{F I X}, Y_{F I X}$ are plotted and tabulated orderly from " 1 to 4 ", i.e. line 1 illustrates $X(t), 2$ is $Y(t)$, line 3 illustrates $X_{F I X}, 4$ is $Y_{F I X}$ [Figure A3]. Where we did apply the coordinate change, where the index [FIX] corresponds to the non rotating inertial system in which VOYAGER SC operate. And we know that using the transpose to the transformation written in the Equations above, we get the values of the field in the inertial system, i.e., FIX coordinate system (see e.g. Goldstein, 1951). And we proceed first to obtain the value of the constant $f=\omega /(2 \pi)$ to evaluate the transformation needed, see Figure A2.

Figure $\mathrm{A} 4(\mathrm{~A})$ shows the measurement at MAGROL, blue line are $48 \mathrm{~s}$ ave values along $X \mathrm{PL}$, IN magnetometer, in counts relative to identified value of off-set of the (B) field. Red-line is synthetic data for a fix-amplitude and identified periodas illustrated in Figure A2.

Plots in Figures A4 clearly show that it was naïve then to assume that the rotation takes place at constant frequency. At least this is strictly the case in the specific MAGROL we attempted trivially using the transform from the above Equations to evaluate the (B) field strength along the inertial frames of $X \mathrm{PL}$, and $Y$ PL. 


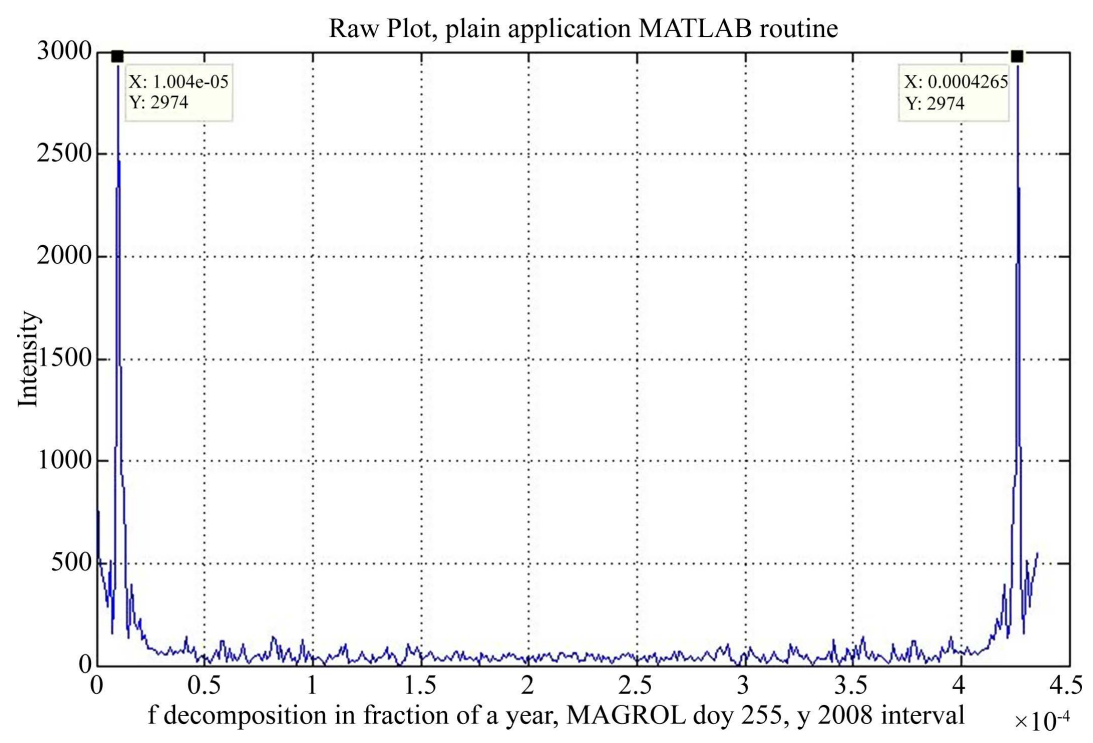

Figure A2. Intensity as a determination of frequency of rotation for the MAGROL interval shown in Figure A1.

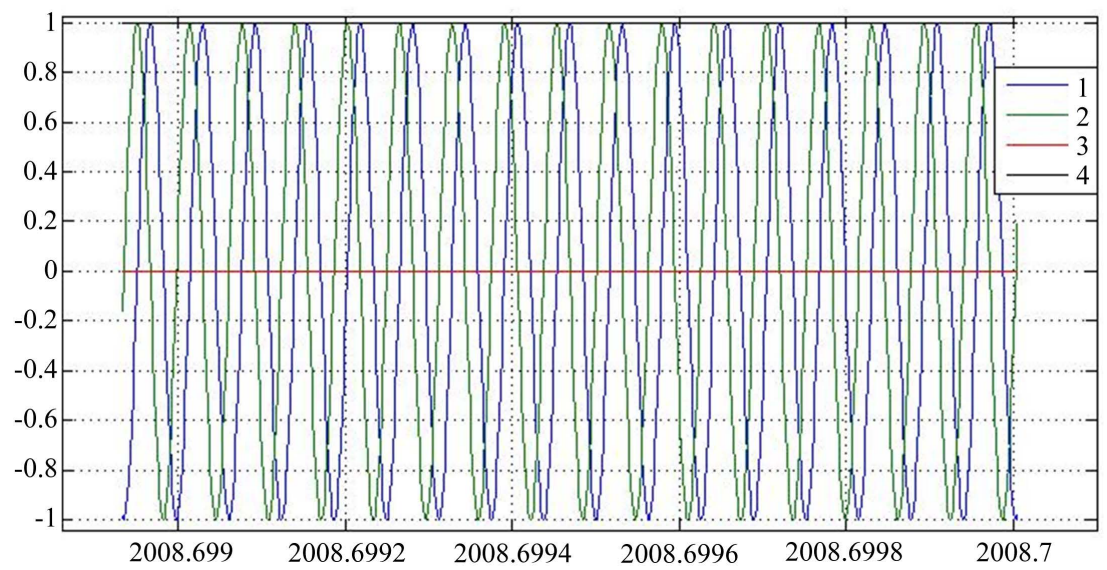

Figure A3. Synthetic data generated both in fix and rotating coordinate system, see body of the text for a complete description.

Another technical detail to consider is that we first were able to identify only eight rotations but a more careful analysis discovered that due to an intensity at the range of the apparent noise in the data we first failed to notice that we actually had ten rotations. In the final adjustment that fact was taken into consideration, i.e., we had in this MAGROL a total of ten rotations.

So the transformation we used fails to provide the values for the inertial directions labeled here as $X \mathrm{PL}$ and $Y \mathrm{PL}$. This is illustrated in Figures A5.

In Figure A5 we identify the following: Line 1, solid blue, is $X$ PL during MAGROL, line 2, solid green, is $Y$ PL during MAGROL, line 3, solid purple, transformation to $X$ fix, solid 4, transformation to $Y$ fix, solid 5 to 7 attempts changing amplitude and frequency to achieve the fix coordinate value of $X$ and $Y$ PL, through frequency small variation, and phase representing a subset of all attempts made. 


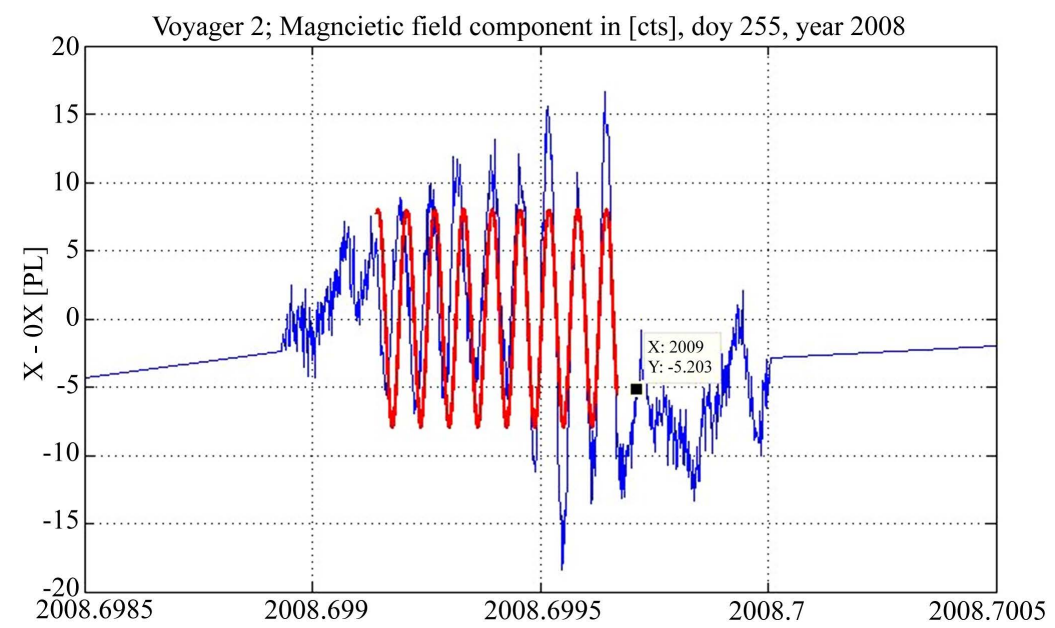

(A)

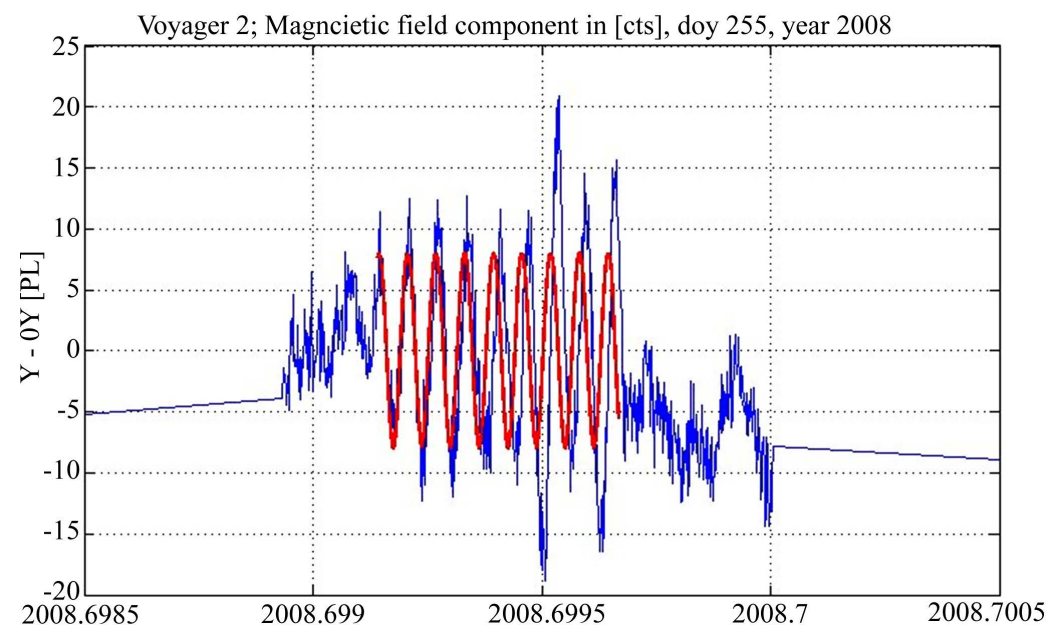

(B)

Figure A4. (A) Data in blue, and in red the phase shift identified and used also in Figure 3; (B): Ibid (A), but this is for the IN sensor along YPL direction.

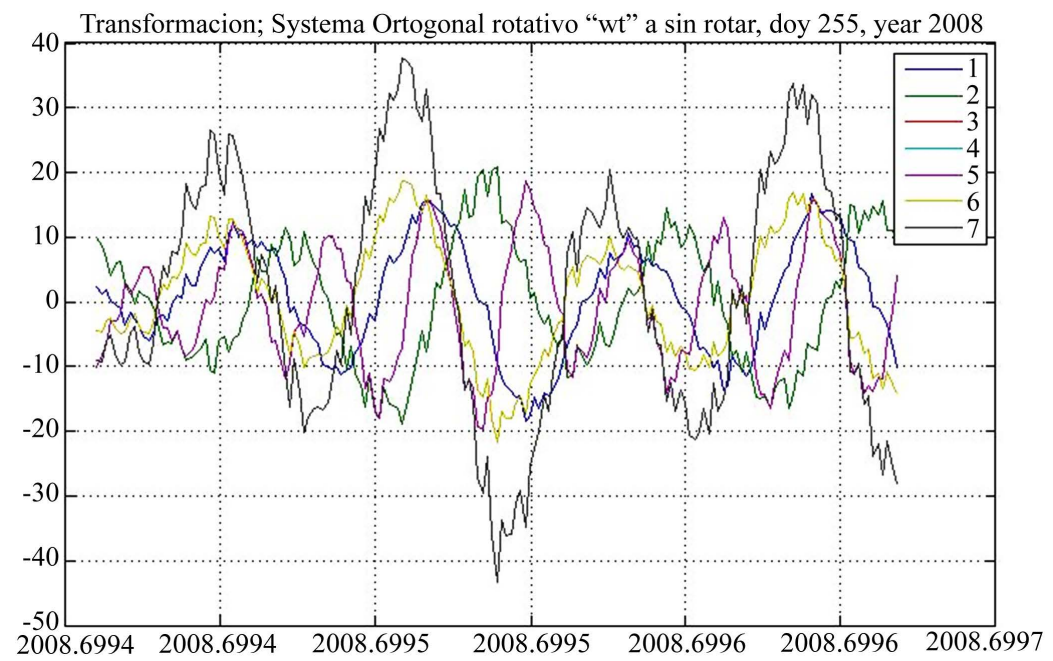

Figure A5. Illustrated is an attempt to correct phase shift and rotational period to reach the stationary frame from the MAGROL rotating $X$ and $Y$ PL sensors. 
Figure A5 illustrates our inability to obtain the inertial value in the case of the $Y$ PL direction. Blue and green curves correspond to $X(t)$ and $Y(t)$. The other color coded lines are attempts to achieve the transformation and the optimal solution which is the one obtained with the black curve (line 7).

With Figure A6 we illustrate using a synthetic imitation of rotating frame like it happens for the period of $38 \mathrm{~min}$ for $X \mathrm{PL}$ and $\pi / 2$ phase shift as it would correspond for $Y$ PL (solid green) and a slightly shifted frequency for the transformation represented by the solid purple line.

With the help of the analysis illustrated in Figure A6 we find that part of our failure is that to reach the the inertial frame we cannot use the well known simple transformation [17]. Figure A6 we tells that when we proceed to modify the frequency in the modeled transformation by $1 / 8$ th of the correct " $\mathrm{f}$ " value it is found a transformation that fail to work simply because in that case we are transforming from rotation with $\omega$ to one that still is not inertial but at a much lower speed of rotation. This reminds us of our curve number 7 solution illustrated in Figure A5.

We concluded that the situation is even more complicated and suspect that in this case we may be confronted with a slow time dependence of the rotation during the duration of the MAGROL. Hence, we proceeded to consider the evolution at each location at completion of a period of rotation consistent with the time before and after termination of MAGROL, We also took additional consideration of the placement of the mean smoothed value of the observed rotation for both axes. This laborious process allowed us to identify at a specific location the likely value of the field for the inboard at $X \mathrm{PL}$ and at $Y \mathrm{PL}$.

The following figures show the painstaking process of determining the value of B-field along $X_{P L}$ and $Y_{P L}$. using the aforementioned technique.

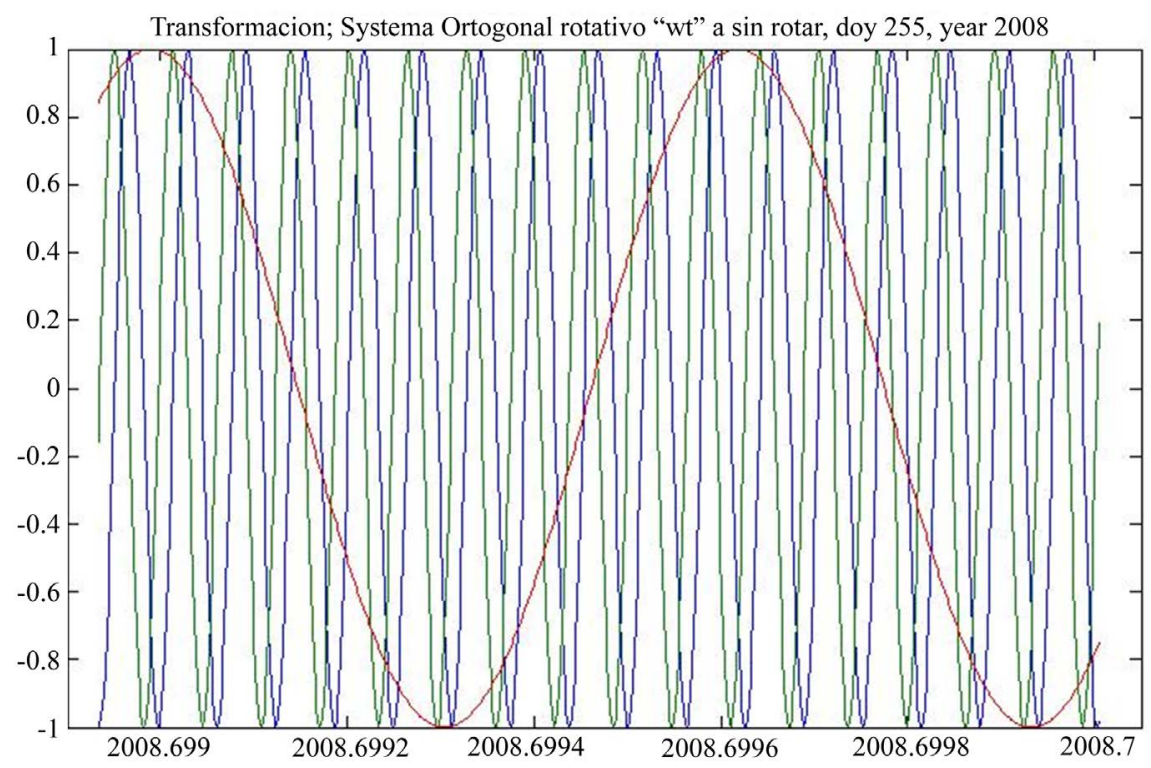

Figure A6. A model simplified representation of the conditions encountered in our analyses. 
Figure A7(A) illustrates with solid blue line is $X$ PL, synthetic data represented at constant amplitude by solid green line. Red-star are the identified zero at mean value for each one of the ten rolls. Solid turquoise thick line intensity of the recovered Figure A7 B-field component along $X$ PL stationary orientation of the SC.

In Figure A7 line 1 gives the data at MAGROL, line 2 gives the frequency identified through the FFT with the same arbitrary amplitude of previous figures. Symbol * gives the center of each roll value, the canonical location of the zero of the micro interval. Line 4 gives therefore the value at that location of the magnetic field.

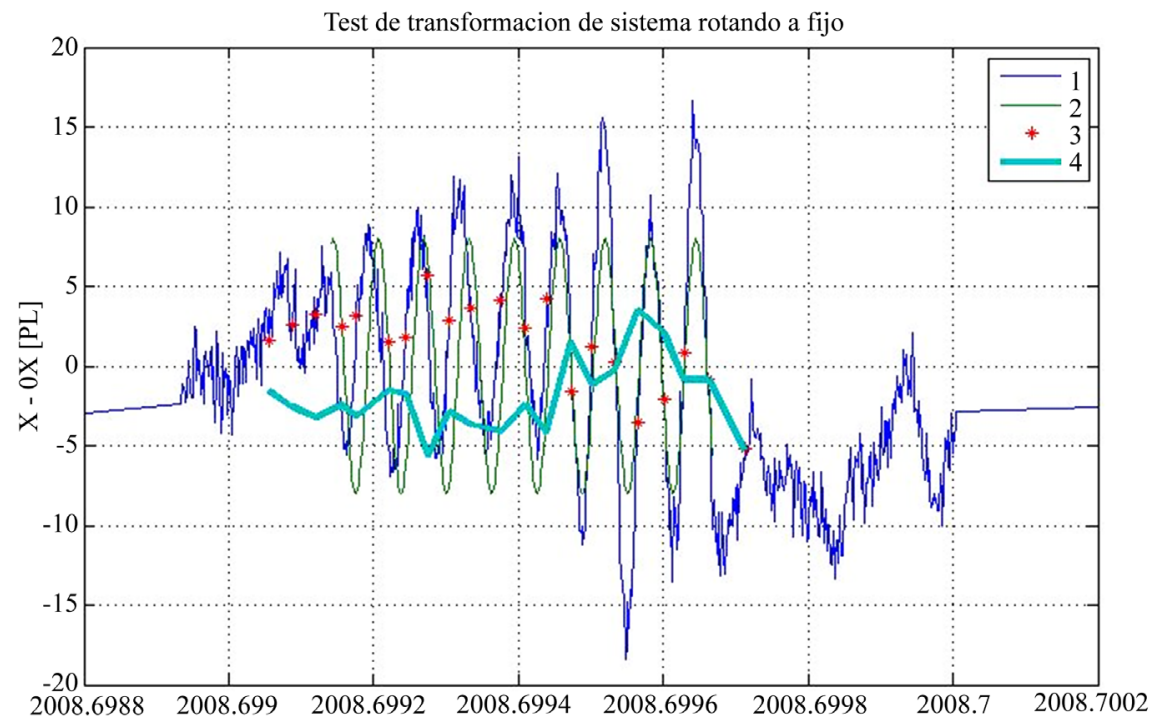

(A)

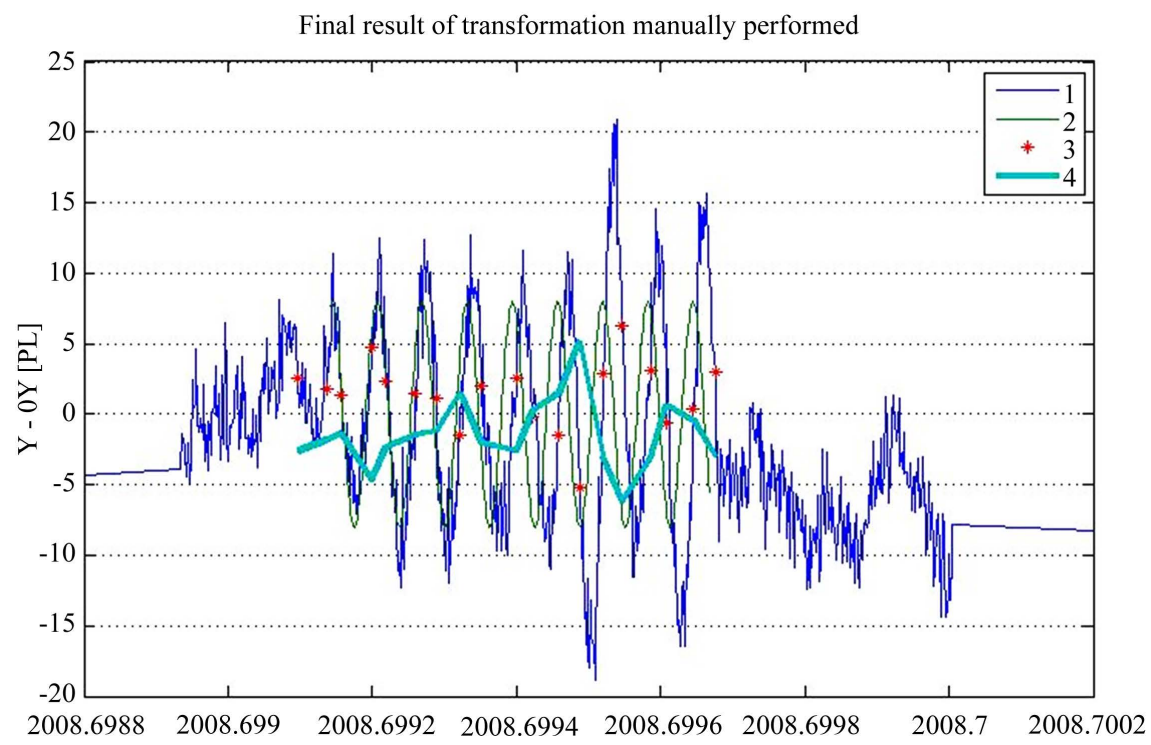

(B)

Figure A7. (A): Our scheme to extract stationary $X$ PL intensity of the B-field at MAGROL; (B): ibid (A) but for the $Y$ PL stationary orientation of the SC. 


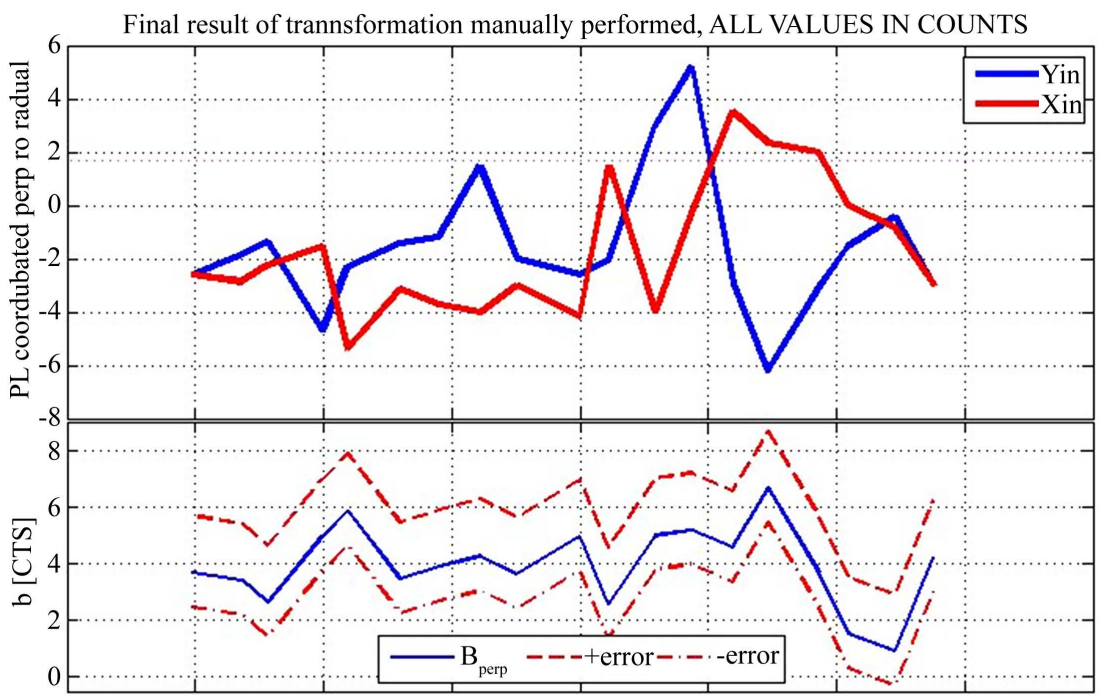

2008.6992008 .69912008 .69922008 .69932008 .69942008 .69952008 .69962008 .69972008 .6998

Figure A8. Illustration of the recovered B-field components at MAGROL along stationary $X$ and $Y$ PL.

Figure 8 constitutes a presentation of the derived B-field in inertial frame. i.e. $\mathrm{X}_{\mathrm{FIX}}$. $\mathrm{Y}_{\mathrm{FIX}}$.

In Figure A8 we show the following: In top panel the recovered $X$ and $Y \mathrm{PL}$ orientation as a function of the time. Bottom panel shows the derived perpendicular B-field to radial from the Sun component at MAGROL. 